\title{
Attenuation of lung fibrosis in mice with a clinically relevant inhibitor of glutathione-S-transferase $\pi$
}

David H. McMillan, ${ }^{1}$ Jos L.J. van der Velden, ${ }^{1}$ Karolyn G. Lahue, ${ }^{1}$ Xi Qian, ${ }^{1}$ Robert W. Schneider, Martina S. Iberg, ${ }^{1}$ James D. Nolin, ${ }^{1}$ Sarah Abdalla, ${ }^{1}$ Dylan T. Casey, ${ }^{1}$ Kenneth D. Tew, ${ }^{2}$ Danyelle M. Townsend, ${ }^{2}$ Colin J. Henderson, ${ }^{3}$ C. Roland Wolf, ${ }^{3}$ Kelly J. Butnor, ${ }^{1}$ Douglas J. Taatjes, ${ }^{1}$ Ralph C. Budd, ${ }^{4}$ Charles G. Irvin, ${ }^{4}$ Albert van der Vliet, ${ }^{1}$ Stevenson Flemer, ${ }^{5}$ Vikas Anathy, ${ }^{1}$ and Yvonne M.W. Janssen-Heininger ${ }^{1}$

'Department of Pathology and Laboratory Medicine, University of Vermont, Burlington, Vermont, USA. 'Department of Cell and Molecular Pharmacology, Medical University of South Carolina, Charleston, South Carolina, USA. ${ }^{3}$ Division of Cancer Research, University of Dundee, Dundee, United Kingdom. ${ }^{4}$ Department of Medicine and ${ }^{5}$ Department of Chemistry, University of Vermont, Burlington, Vermont, USA.

Idiopathic pulmonary fibrosis (IPF) is a debilitating lung disease characterized by excessive collagen production and fibrogenesis. Apoptosis in lung epithelial cells is critical in IPF pathogenesis, as heightened loss of these cells promotes fibroblast activation and remodeling. Changes in glutathione redox status have been reported in IPF patients. S-glutathionylation, the conjugation of glutathione to reactive cysteines, is catalyzed in part by glutathione-S-transferase $\pi$ (GSTP). To date, no published information exists linking GSTP and IPF to our knowledge. We hypothesized that GSTP mediates lung fibrogenesis in part through FAS S-glutathionylation, a critical event in epithelial cell apoptosis. Our results demonstrate that GSTP immunoreactivity is increased in the lungs of IPF patients, notably within type II epithelial cells. The FAS-CSTP interaction was also increased in IPF lungs. Bleomycin- and AdTCF $\beta$-induced increases in collagen content, $\alpha$-SMA, FAS S-glutathionylation, and total protein S-glutathionylation were strongly attenuated in $\mathrm{Gstp}^{-/}$- mice. Oropharyngeal administration of the GSTP inhibitor, TLK117, at a time when fibrosis was already apparent, attenuated bleomycin- and AdTCF $\beta$-induced remodeling, $\alpha$-SMA, caspase activation, FAS S-glutathionylation, and total protein S-glutathionylation. CSTP is an important driver of protein S-glutathionylation and lung fibrosis, and GSTP inhibition via the airways may be a novel therapeutic strategy for the treatment of IPF.

Conflict of interest: The authors have declared that no conflict of interest exists.

Submitted: November 25, 2015 Accepted: May 4, 2016 Published: June 2, 2016

Reference information: JCI Insight. 2016;1(8):e85717. doi:10.1172/jci.insight.85717.

\section{Introduction}

Pulmonary fibrosis is a debilitating disease characterized by the development of excess fibrous tissue, leading to thickening of the alveolar walls and diminished lung function (1). Idiopathic pulmonary fibrosis (IPF), the most common subtype of interstitial lung disease, affects more than 120,000 Americans and claims approximately 40,000 lives each year $(2,3)$. No cure exists, current treatment options are limited, and the average life expectancy upon diagnosis is 3 to 5 years (2-4). In normal settings, injury of the epithelium is counterbalanced by repair mechanisms that restore normal epithelial structure and function (5). In cases of fibrosis, however, this balance is overwhelmed and excessive epithelial damage occurs (5). Chronic injury of the distal airways and subsequent loss of the bronchiolar and alveolar epithelial cells, a common histopathological feature of IPF, occurs concurrently with the activation and overproliferation of myofibroblasts $(5,6)$. Lung epithelial cell apoptosis also promotes increased release of TGF- $\beta$, a crucial mediator of fibrotic remodeling (7), and loss of these cells is sufficient to drive fibrosis (8). Previous studies from our laboratory and others have demonstrated that FAS, a proapoptotic member of the TNF receptor superfamily, is a crucial mediator in the pathogenesis of fibrosis $(6,8)$. Activation of FAS, by binding of FAS ligand (FASL), promotes apoptosis of bronchiolar and alveolar epithelial cells through caspase-3 and -8 (8). Myofibroblasts from IPF patients express FASL and induce FAS-dependent apoptosis in lung epithelial cells, promoting an imbalance between these cell types (9). 
FAS-mediated apoptosis is dependent on a variety of factors, including changes in redox status (10). Glutathione (GSH), a thiol-containing tripeptide, is a highly abundant endogenous antioxidant and functions, in part, as a free radical scavenger (10). Decreases in GSH and increases in GSH disulfide, the oxidized form of GSH, have been reported in IPF patients $(10,11)$. GSH can also regulate protein structure and function through a process known as S-glutathionylation, the reversible conjugation of a GSH molecule to reactive cysteine residues in proteins (10). Our group has recently demonstrated that FASL binding promotes the S-glutathionylation of cysteine 294 of FAS in mouse airway epithelial cells and that this modification promotes cell surface expression of FAS and activation of the caspase cascade $(8,12)$. The exact mechanisms that lead to protein S-glutathionylation (PSSG) in pathobiological settings are not fully understood, but it is believed that oxidants such as hydrogen peroxide can oxidize sulfhydryl groups of protein cysteine residues to form sulfenic acid intermediates, which are subsequently S-glutathionylated (10).

S-glutathionylation can occur nonenzymatically by directly binding free GSH under conditions of high oxidative stress, but these reactions are believed to be fairly nonspecific and are associated with toxicity and cell death (13). Enzymatic PSSG, however, is highly specific and tightly controlled by a variety of enzymes, including glutathione- $S$-transferases (GSTs), a class of enzymes critical in phase II drug metabolism (14, 15). GST $\pi$ (GSTP), which is expressed primarily in the lung, brain, and gastrointestinal tract (15), is a mediator of S-glutathionylation in lung airway epithelial cells $(5,8,10)$ and has been linked to both asthma and lung cancer $(16,17)$.

Despite the aforementioned links between GSTP and FAS S-glutathionylation (FAS-SSG), no published reports exist to date on the role of GSTP-linked PSSG during lung fibrogenesis. Likewise, the effects of either GSTP inhibition or ablation on fibrotic remodeling have not yet been determined. Herein, we determined that both global knockout and pharmacological inhibition of GSTP, using a clinically relevant inhibitor, attenuate fibrotic remodeling in two mouse models of pulmonary fibrosis, in part, through a mechanism involving FAS-SSG. Our findings presented herein suggest that GSTP inhibition may be a novel target for the treatment of IPF and other lung fibroproliferative diseases.

\section{Results}

GSTP immunoreactivity and FAS-GSTP interaction are increased in the lungs of IPF patients. We first determined whether GSTP expression was affected in the lungs of IPF patients. While control tissues exhibited sparse weak GSTP immunoreactivity in pneumocytes (Figure 1A; 4 top left images) and weak intensity staining in bronchial airway epithelium (Figure 1A; top rightmost image), strong staining was identified in IPF lung tissue within reactive type 2 pneumocytes in areas of active fibrosis (Figure 1A; middle and bottom). These areas, which were at the interface of densely fibrotic and less affected lung tissue, featured loose fibrous tissue and fibroblast foci. Marked increases in GSTP immunoreactivity were readily detectable in IPF lung tissues in regions of bronchiolar metaplasia in honeycomb cysts (Figure 1B). GSTP-reactive cells in the latter area also expressed pro-surfactant protein C (pro-SPC), a marker of type II alveolar epithelial cells (Figure 1B). Based on our prior studies demonstrating that GSTP interacts with FAS in the lungs of bleomycin-treated mice (8), we next assessed whether a similar interaction between GSTP and FAS was present in the lungs of IPF patients. Results in Figure 1C demonstrate an interaction between GSTP and FAS in 5 of 10 IPF patients, while little to no interaction was observed in the control lung tissues. Similarly, using two independent mouse models of lung fibrosis, bleomycin or a TGF- $\beta 1$-expressing recombinant adenovirus (AdTGF $\beta$ ), we demonstrated increases in GSTP immunoreactivity in lung tissues 2 weeks after administration of either agent, with increases being apparent in bronchiolar epithelium and distal lung regions. Increased GSTP immunoreactivity was concurrent with the onset of fibrotic remodeling (ref. 18 and Figure 1D).

Gstp deficiency attenuates bleomycin- and AdTGFß-induced collagen content and fibrotic remodeling. To determine the importance of GSTP in the pathogenesis of lung fibrosis, we next subjected Gstp ${ }^{-/}$mice (Supplemental Figure 1; supplemental material available online with this article; doi:10.1172/jci.insight.85717DS1) and their wild-type counterparts to either the bleomycin or AdTGF $\beta$ models of fibrosis. As expected in wild-type mice, hydroxyproline and soluble collagen content were significantly increased at 15 and 28 days following bleomycin administration. In contrast, bleomycin-induced soluble collagen content was significantly attenuated in the Gstp ${ }^{-/-}$mice (Figure 2, A and B, and Supplemental Figure 2). Although downward trends in hydroxyproline content were observed in bleomycin-treated Gstp ${ }^{-/}$mice relative to controls, these trends did not achieve statistical significance (Figure 2A). Assessment of collagen deposition by Masson's trichrome staining (Figure 2C) confirmed that bleomycin-induced increases in fibrotic remodeling were 

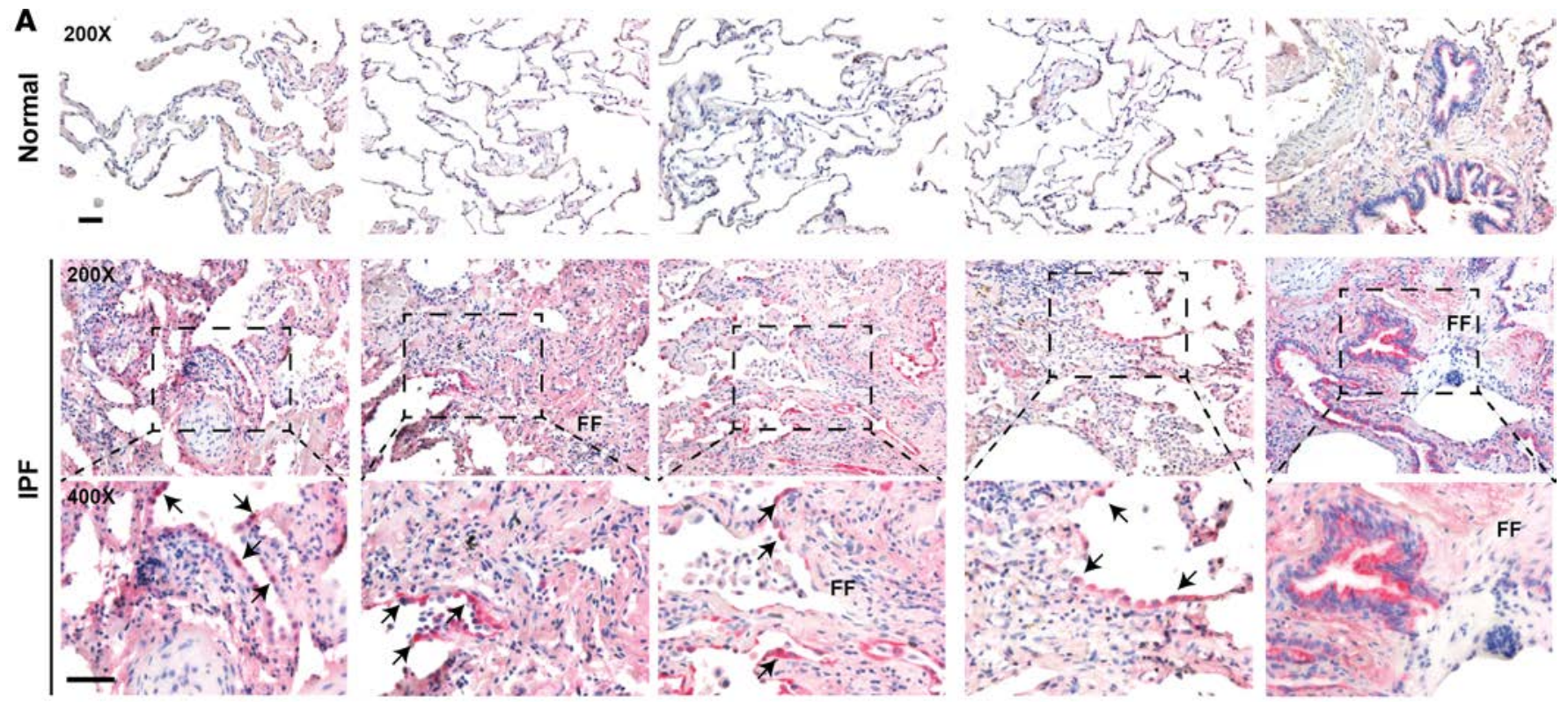

B GSTP1/pro-SPC expression in reactive type II pneumocytes

D

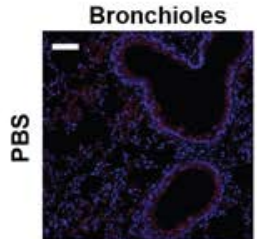

Parenchyma
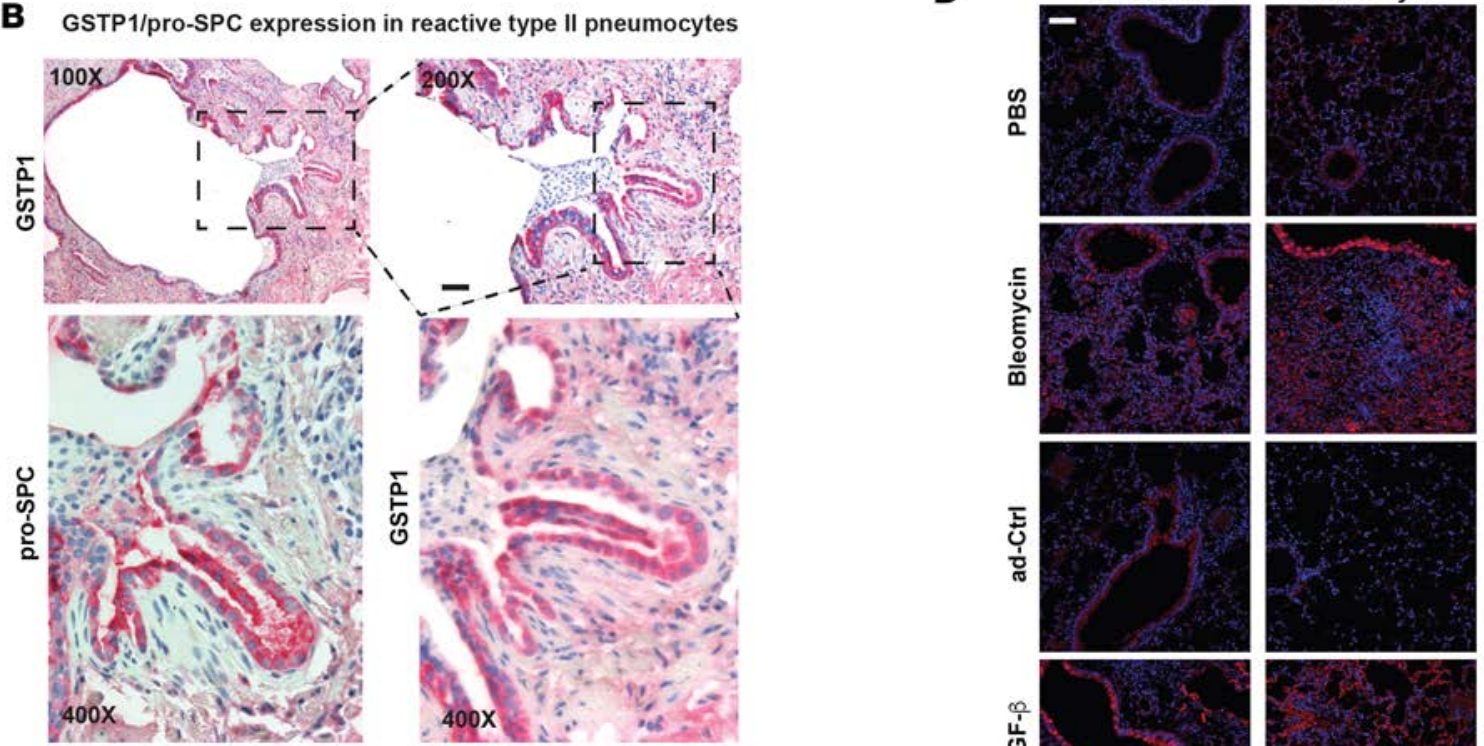

\section{C}
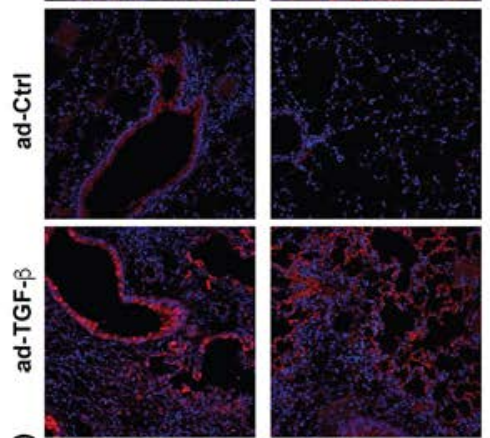

IP: Fas

Normal

?

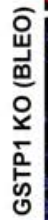

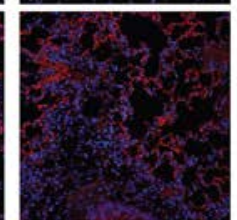

WB: GSTP

WB: Fas

- - - - - - - -
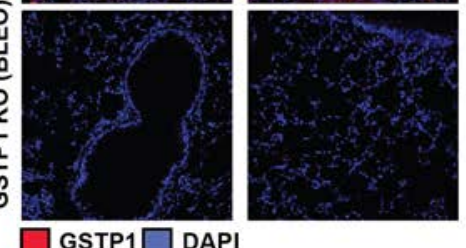

GSTP1 $\square$ DAPI

Figure 1. GSTP immunoreactivity and FAS-CSTP interaction are increased in the lungs of IPF patients. (A) Evaluation of GSTP immunoreactivity in human lung sections from either control or IPF patients (red, GSTP; blue, hematoxylin). Representative images of lung sections from 5 of 14 patients from each group are shown. Arrows indicate reactive type 2 pneumocytes. Scale bar: $50 \mu \mathrm{m}$. (B) Assessment of pro-SPC immunoreactivity in lung sections from IPF patients. Parallel sections from IPF patients were probed for either CSTP or pro-SPC by immunohistochemistry (red, GSTP or pro-SPC as indicated; blue, hematoxylin). Scale bar: $50 \mu \mathrm{m}$. (C) Assessment of interaction between CSTP and FAS in the lungs of IPF patients. FAS was isolated by immunoprecipitation from whole-lung homogenates of both normal and IPF patients, and GSTP was detected by Western blot. Lanes represent individual patients. (D) Evaluation of GSTP immunoreactivity in mouse lungs treated with bleomycin for 15 days or AdTCF $\beta$ for 21 days. GSTP was detected by immunohistochemistry (red, GSTP; blue, hematoxylin). Scale bar: $50 \mu \mathrm{m}$. IPF, idiopathic pulmonary fibrosis; FF, fibroblast foci; GSTP, glutathione-S-transferase $\pi$; SPC, surfactant protein C; ad-Ctrl, control adenoviral vector; ad-TGF- $\beta$, adenoviral vector expressing active TGF- $\beta 1$. 


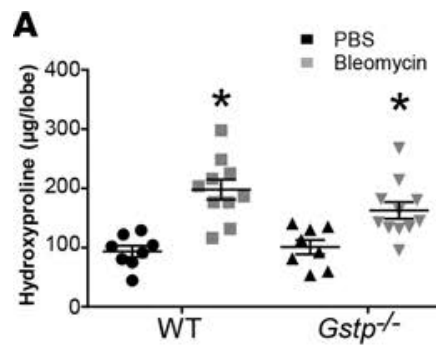

C

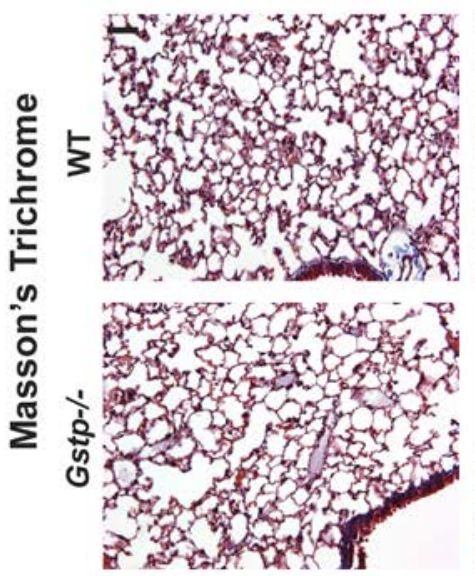

D

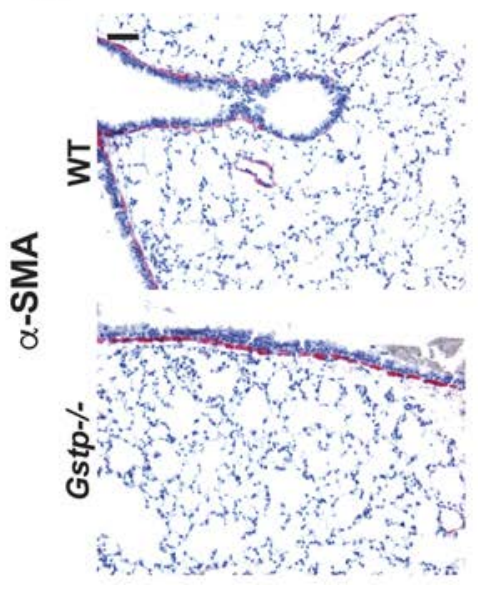

B E

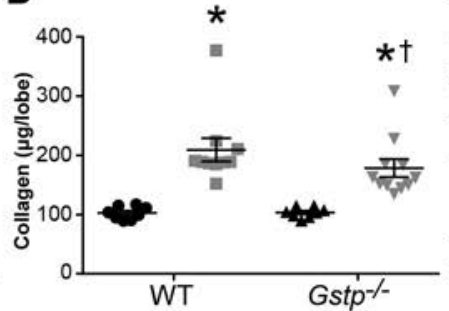

Bleomycin
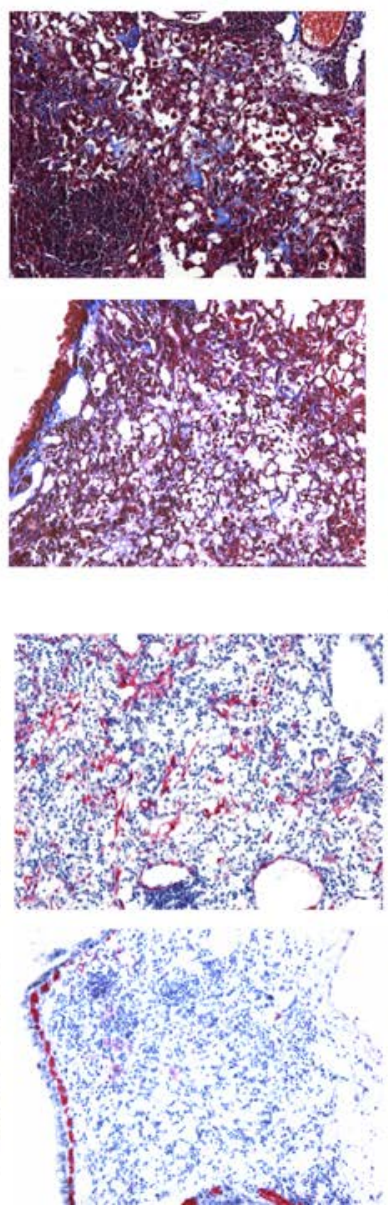

G

AdCtrl
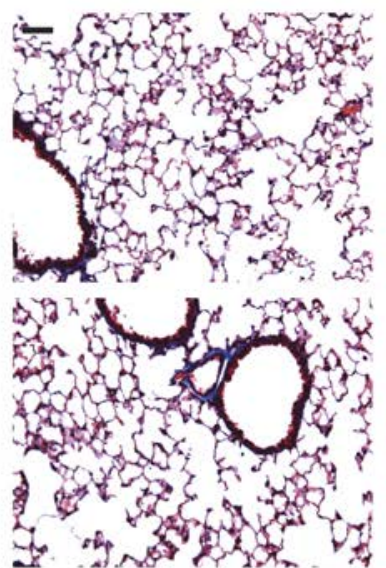

H

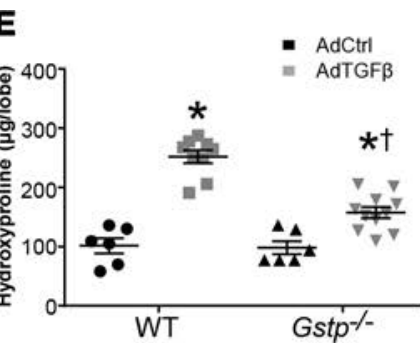

F
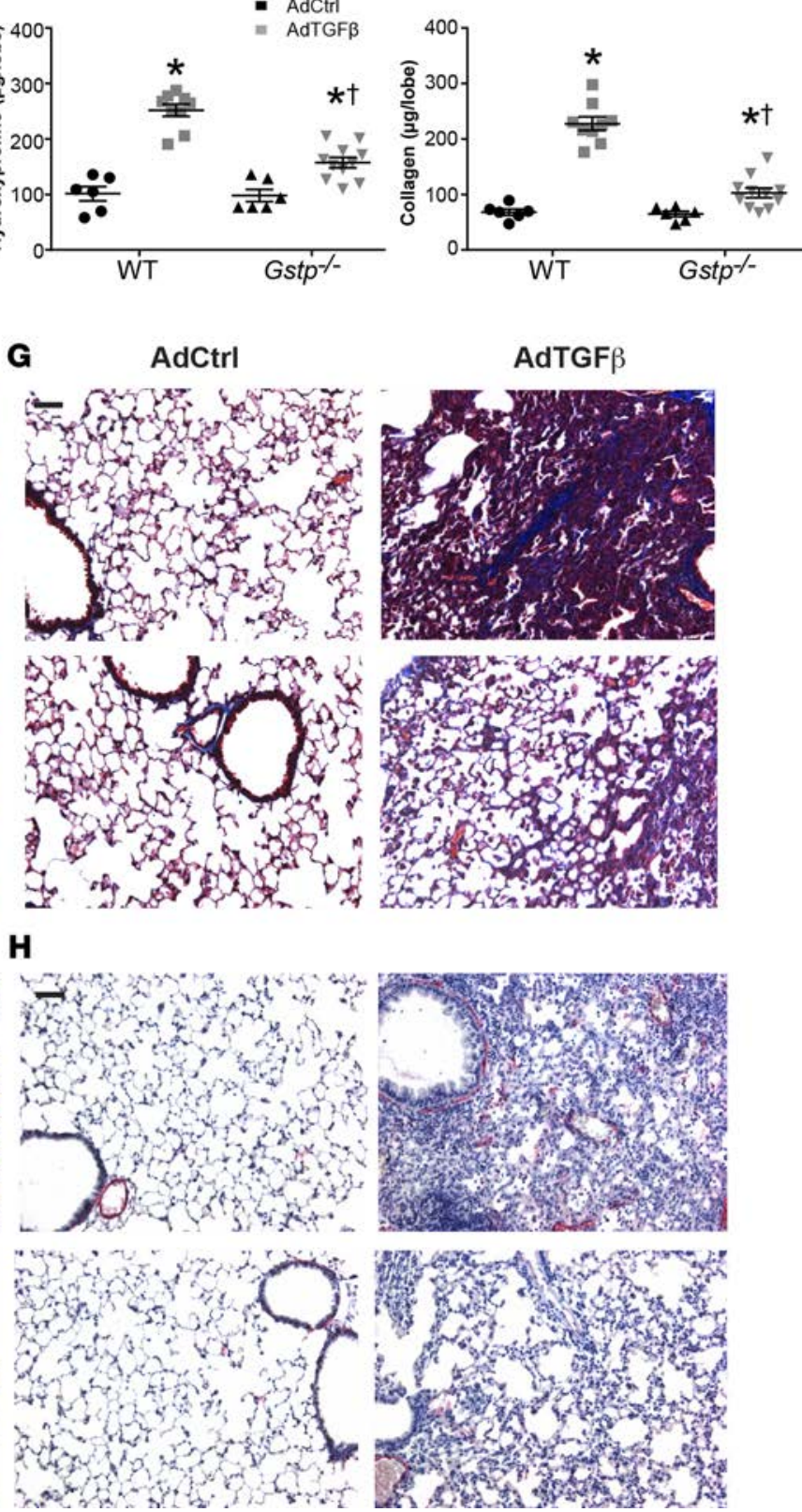

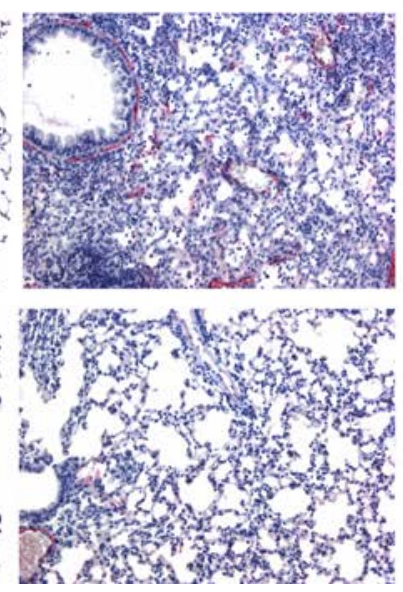

Figure 2. GSTP deficiency attenuates bleomycin- and AdTGF $\beta$-induced collagen content and fibrotic remodeling. Wild-type and Gstp ${ }^{-/-}$mice were treated with (A-D) bleomycin for 15 days or (E-H) AdTCF $\beta$ for 21 days. (A and B) Assessment of hydroxyproline and soluble collagen content in the lungs of wild-type or $\mathrm{Sstp}^{-/-}$mice 15 days after bleomycin administration. (C) Assessment of fibrotic remodeling by Masson's trichrome staining 15 days after bleomycin administration. (D) Detection of $\alpha$-SMA expression by immunohistochemistry (red, $\alpha$-SMA; blue, hematoxylin). (E and F) Assessment of hydroxyproline and soluble collagen content in the lungs of wild-type or Gstp ${ }^{-1-}$ mice 21 days after AdTCF $\beta$ treatment. (G) Assessment of fibrotic remodeling by Masson's trichrome staining 21 days after infection. (H) Assessment of $\alpha$-SMA immunoreactivity by immunohistochemistry (red, $\alpha$-SMA; blue, hematoxylin). ${ }^{*} P<0.05$ relative to PBS or AdCtrl group, ${ }^{\dagger} P<0.05$ relative to respective wild-type group by 2 -way ANOVA with a Tukey post-test. Scale bar: $100 \mu \mathrm{m}$. Shown are pooled data from 2 independent experiments ( $n=6-11$ per group). GSTP, glutathione-S-transferase $\pi ; \alpha$-SMA, $\alpha$ smooth muscle actin; AdCtrl, control adenoviral vector; AdTCF $\beta$, adenoviral vector expressing active TGF- $\beta 1$.

attenuated in Gstp ${ }^{-/-}$mouse lungs. Furthermore, immunoreactivity of $\alpha$ smooth muscle actin ( $\alpha$-SMA), a marker of activated fibroblasts (19), was also noticeably attenuated in the absence of Gstp (Figure 2D). mRNA expression of the mesenchymal and fibrosis markers, collagen type 1A1 and 5A1 (Colla1 and Col5a1), fibroblast-specific protein $1(F s p 1)$, and fibronectin $(F n 1)$, were also increased following bleomycin administration in wild-type mice (Supplemental Figure 3A). These increases were either significantly decreased (Colla1 and Fsp1) or trended toward being decreased (Col5a1 and Fn1) in Gstp ${ }^{-1}$ lungs. 
We next corroborated these observations in a different fibrosis model by evaluating the impact of Gstp deficiency in mice exposed to AdTGF $\beta$. Similar to the bleomycin model, results in Figure 2, E-H, demonstrate that AdTGF $\beta$-mediated increases in hydroxyproline, soluble collagen, Masson's trichrome staining, and $\alpha$-SMA immunoreactivity were strongly attenuated in $G s t p^{-/}$mouse lungs. Last, AdTGF $\beta$-induced Colla1 mRNA expression was significantly decreased in $G s t p^{-/}$lung tissues (Supplemental Figure 3B).

Gstp deficiency attenuates bleomycin- and AdTGFB-induced PSSG. To determine how GSTP may be regulating bleomycin- and AdTGF $\beta$-induced fibrotic remodeling in mouse lungs, we next examined its effects on PSSG in these models. While GSTP has been shown to mediate GSH-conjugation reactions, as it pertains to the metabolism of xenobiotics, its ability to catalyze PSSG in vivo remains unclear (20). In mice exposed to either bleomycin or AdTGF $\beta$, total PSSG was increased in lung tissues (Figure 3, A and B), consistent with previous observations (21). Remarkably, bleomycin- and AdTGF $\beta$-mediated increases in total PSSG were almost completely absent in $G s t p^{-1-}$ mice, demonstrating that GSTP is a strong contributor to PSSG in these models. Our group has previously shown that FAS-SSG is essential in augmenting epithelial cell apoptosis and that FAS is required for the development of fibrosis $(8,12)$. Results in Figure $3, \mathrm{C}$ and D, demonstrate that FAS-SSG was increased in mouse lungs 15 or 21 days after exposure to either bleomycin or AdTGF $\beta$, respectively. In contrast, no increases in FAS-SSG were detected in lung tissues from Gstp ${ }^{-1}$ mice exposed to bleomycin or AdTGF $\beta$. These findings suggest that FAS may be an important target for GSTP-catalyzed PSSG and fibrogenesis.

TLK117-mediated GSTP inhibition attenuates bleomycin- and AdTGFß-induced collagen content. Having determined that absence of Gstp attenuates bleomycin- and AdTGF $\beta$-induced fibrotic remodeling and FASSSG, we next assessed the impact of pharmacological inhibition of GSTP on lung fibrotic remodeling. The active GSTP inhibitor, TLK117, is a GSH analog and is the active metabolite of TLK199, which has completed phase II clinical trials for myelodysplastic syndrome $(22,23)$. TLK117 is the most specific GSTP inhibitor to date, with a binding affinity greater than GSH itself and a selectivity for GSTP over 50-fold greater than the GSTM and GSTA classes (inhibition constant $\left.\left[K_{i}\right]=0.4 \mu \mathrm{M}\right)(15,24-27)$. No published observations on the effects of TLK117 in settings of lung fibrotic remodeling yet exist.

We first explored whether TLK117-mediated inhibition of GSTP can attenuate FASL-induced apoptosis. Exposure to FASL significantly increased caspase-3 activity in wild-type mouse lung fibroblasts and in Fas-deficient ( $l p r$ ) fibroblasts in which wild-type Fas has been reconstituted (Figure 4A and Supplemental Figure 4). TLK117 pretreatment significantly attenuated FASL-induced caspase-3 activity in these groups. In $l p r$ fibroblasts expressing C294A mutant Fas, which is refractory to S-glutathionylation (12), FASL-induced caspase-3 activity was completely attenuated. Further, no effect of TLK117 was observed in these settings, indicating that the ability of TLK117 to attenuate FASL-induced apoptosis requires functional FAS that can be S-glutathionylated. Although TLK117 attenuated FASL-induced caspase-3 activity in type II alveolar epithelial cells, activation of caspase- 3 induced by TNF- $\alpha$ and cycloheximide was not affected by TLK117 (Figure 4B). Collectively, these findings suggest that the protective effects of TLK117-mediated GSTP inhibition are specific to FAS-mediated apoptosis.

To determine the effects of TLK117 in lung fibrosis, we first confirmed that oropharyngeal administration of TLK117 inhibits GSTP activity in lung tissues. Four hours after administration of $50 \mathrm{mg} / \mathrm{kg}$ TLK117, GSTP activity was strongly decreased and remained decreased by about $60 \%$ for at least 24 hours (Figure 4C). A dose of $25 \mathrm{mg} / \mathrm{kg}$ of TLK117, however, did not significantly affect GSTP activity. We therefore used a $50 \mathrm{mg} / \mathrm{kg}$ dosing regimen in subsequent studies. To determine the effect of TLK117-mediated GSTP inhibition on preexisting fibrotic remodeling in the bleomycin model, TLK117 was administered at $50 \mathrm{mg} / \mathrm{kg}$ once every 3 days, starting 14 days after bleomycin administration up to day 26, and mice were euthanized on day 28. Mice that had received either bleomycin or PBS concurrently were euthanized on day 15 to confirm existence of remodeling at the onset of TLK117 treatment (Figure 4D). As demonstrated in Figure 4, E and F, bleomycin-induced increases in hydroxyproline and soluble collagen content were significantly increased at day 15 , and both parameters were further increased by day 28 . Importantly, TLK117-mediated GSTP inhibition significantly attenuated the increase in fibrosis between 15 and 28 days. No statistically significant differences in hydroxyproline and soluble collagen content were detected between the bleomycin-exposed mice that received TLK117 and PBS controls. Masson's trichrome staining confirmed that bleomycin-induced lung fibrotic remodeling was markedly decreased upon TLK117-mediated inhibition of GSTP (Figure 4G). Similarly, bleomycin-induced $\alpha$-SMA immunoreactivity was strongly attenuated upon TLK117 treatment (Figure 4H). 

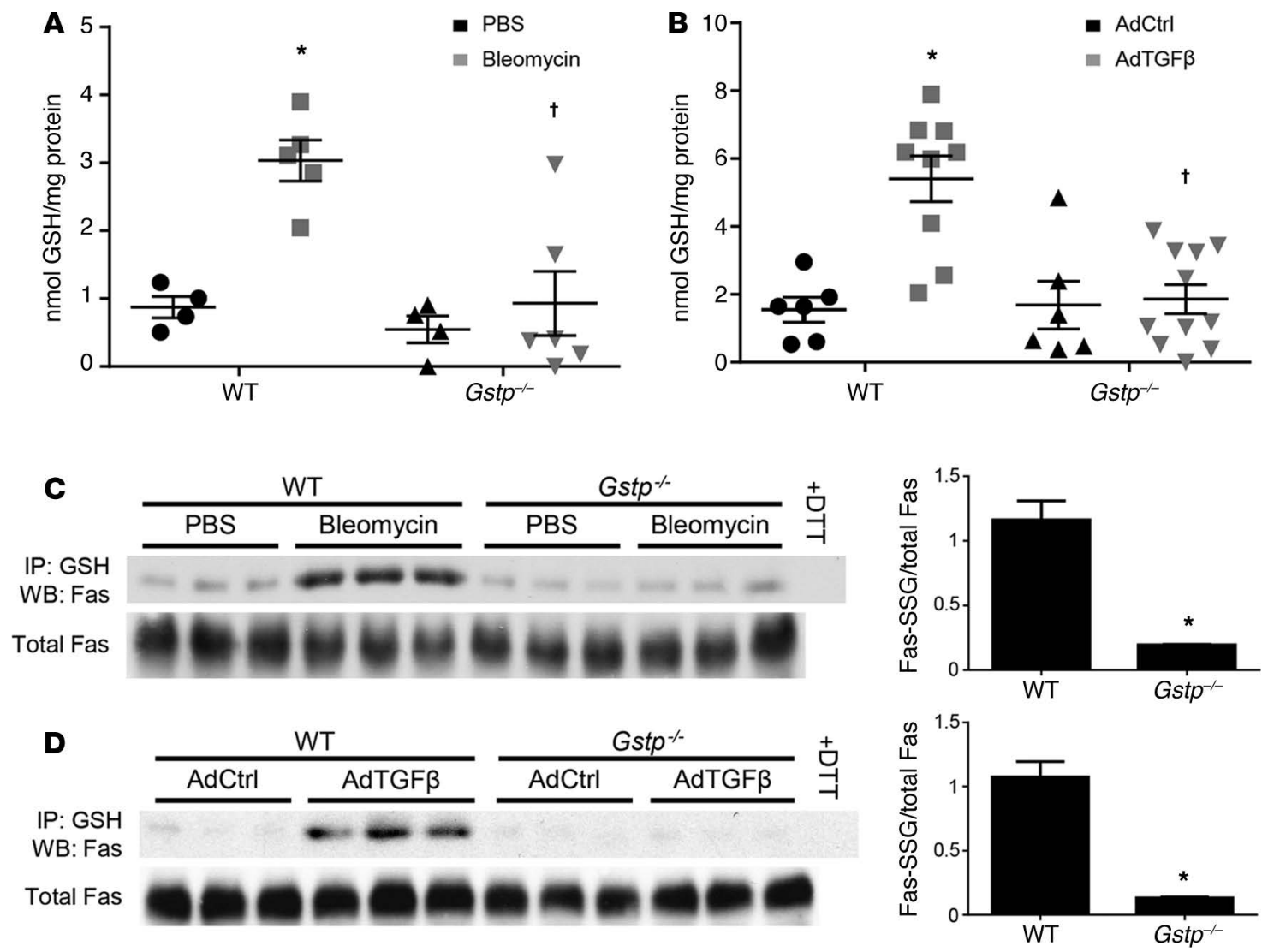

Figure 3. GSTP deficiency attenuates bleomycin- and AdTGF $\beta$-induced protein S-glutathionylation. Wild-type and Gstp ${ }^{-/-}$mice were treated with either bleomycin for 15 days or AdTCF $\beta$ for 21 days as indicated. (A and B) Detection of total PSSG content in mouse lung homogenates with a modified DTNB assay. Shown are pooled data from 2 independent experiments. ${ }^{*} P<0.05$ relative to PBS or AdCtrl group, ${ }^{\dagger} P<0.05$ relative to respective wild-type group by 2-way ANOVA with a Tukey post-test $(n=4-11$ per group). (C and D) Assessment of FAS-SSG content by immunoprecipitation of total PSSG and Western blot for FAS. Statistically significant differences between wild-type and $\mathrm{Gstp}^{-/-}$groups treated with either (C) bleomycin or (D) AdTCF $\beta$ were determined by densitometric analysis. ${ }^{*} P<0.05$ by unpaired Student's $t$ test ( $n=3$ per group). The data shown in $\mathbf{C}$ and $\mathbf{D}$ are representative of 1 of 2 independent experiments. GSTP, glutathione-S-transferase $\pi$; FAS-SSG, S-glutathionylated FAS; AdCtrl, control adenovirus; AdTCF $\beta$, recombinant adenovirus expressing active TCF- $\beta 1$; $C S H$, glutathione; DTT, dithiothreitol; WB, Western blot.

To corroborate these findings, a similar TLK117 regimen was used in the AdTGF $\beta$ model (Figure $5 \mathrm{~A})$. As expected, hydroxyproline and soluble collagen content were significantly increased 14 days after AdTGF $\beta$ infection with further increases occurring by day 28. Administration of TLK117 between days 14 and 28 significantly attenuated AdTGF $\beta$-mediated increases in hydroxyproline, soluble collagen content, Masson's trichrome reactivity, and $\alpha$-SMA immunoreactivity (Figure 5, B-E).

TLK117-mediated GSTP inhibition attenuates caspase activation, PSSG, and FAS-GSTP interaction. To determine whether TLK117-mediated GSTP inhibition affects bleomycin- and AdTGF $\beta$-induced apoptosis in mouse lungs, we measured caspase- 3 and -8 activity in whole-lung homogenates from these mice. Activation of the caspase cascade is a crucial event in apoptosis, and we have previously linked it to FAS-SSG in type II alveolar epithelial cells (8). Caspase-3 and -8 activities were increased both 15 and 28 days after bleomycin treatment and were significantly decreased upon TLK117-induced GSTP inhibition (Figure 6, A and B). Bleomycin-induced total PSSG was also significantly decreased in mouse lungs upon GSTP inhibition (Figure 6C). Further, bleomycin-induced increases in FAS-SSG were significantly attenuated upon TLK117 treatment (Figure 6D).

Similar to the bleomycin model, AdTGF $\beta$-mediated increases in caspase-3 and -8 activity, total PSSG, and FAS-SSG were strongly attenuated upon GSTP inhibition (Figure 7, A-D). Last, we determined whether TLK117 affects the interaction of GSTP and FAS. Results in Figure 7E demonstrate that FASGSTP association, noticeably increased 14 days after AdTGF $\beta$ infection, was significantly decreased by TLK117-mediated GSTP inhibition. 

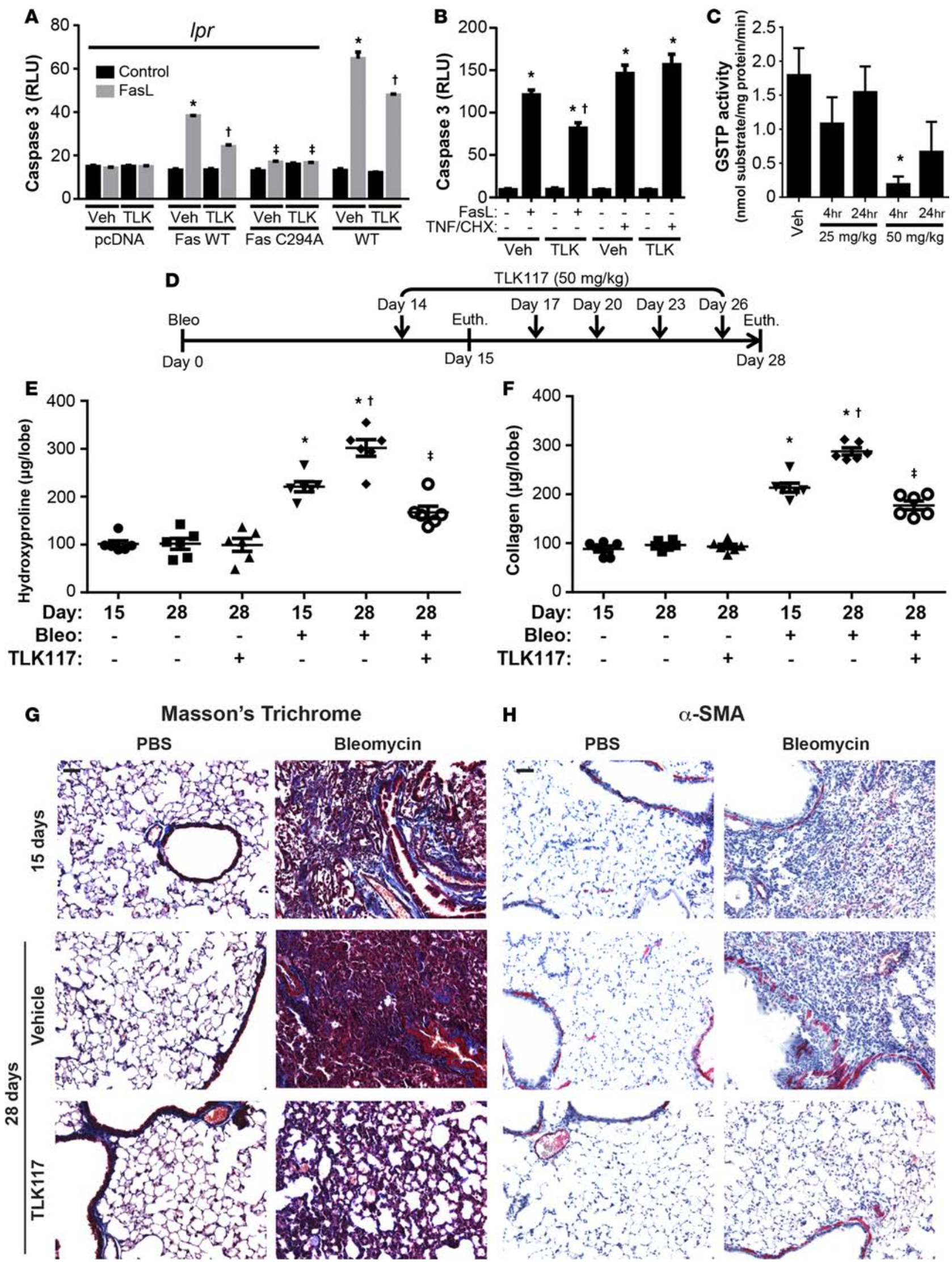
Figure 4. TLK117-induced GSTP inhibition attenuates bleomycin-induced collagen content. (A) Assessment of caspase-3 activity in wild-type and Ipr mouse lung fibroblasts transfected with pcDNA3.1, wild-type Fas, or C294A mutant Fas; pretreated with TLK117 for 2 hours; and treated with FASL for 4 hours. ${ }^{*} P<0.05$ relative to respective vehicle/control group, ${ }^{\dagger} P<0.05$ relative to respective vehicle/FASL group, $\ddagger P<0.05$ relative to respective wild-type FAS construct group by 1-way ANOVA with a Tukey post-test ( $n=3$ per group, representative of 2 independent experiments). (B) Assessment of caspase-3 activity in mouse type II alveolar epithelial cells following TLK117 pretreatment for 2 hours and treatment with either FASL or TNF- $\alpha$ with cycloheximide for 4 hours. ${ }^{*} P<0.05$ relative to respective vehicle/control group, ${ }^{\dagger} P<0.05$ relative to respective vehicle/FASL group by 1 -way ANOVA with a Tukey post-test ( $n=6$ per group, representative of 2 independent experiments). (C) Evaluation of CSTP activity in mouse lungs following oropharyngeal administration of either 25 or $50 \mathrm{mg} / \mathrm{kg}$ TLK117 for 4 or 24 hours. ${ }^{*} P<0.05$ relative to vehicle control by 1 -way ANOVA ( $n=3$ per group). (D) Schematic of the TLK117 treatment strategy with the bleomycin model. (E and F) Assessment of hydroxyproline and soluble collagen content in mouse lung homogenates. ${ }^{*} P<0.05$ relative to PBS group, ${ }^{\dagger} P<0.05$ relative to 15 -day bleomycin group, $¥ P<0.05$ relative to 28 -day bleomycin/vehicle group by 1-way ANOVA with a Tukey post-test. Shown are pooled data from 2 independent experiments ( $n=6$ per group). (G) Assessment of fibrotic remodeling by Masson's trichrome staining. (H) Assessment of $\alpha$-SMA immunoreactivity by immunohistochemistry (red, $\alpha$-SMA, blue, hematoxylin). Scale bar: 100 $\mu \mathrm{m}$. Ipr, Fas-deficient; CSTP, glutathione-S-transferase $\pi$; $\alpha$-SMA, $\alpha$-smooth muscle actin; Bleo, bleomycin; Veh, vehicle; TLK, TLK117; FASL, FAS ligand TNF, TNF- $\alpha$; CHX, cycloheximide; RLU, relative luminescence units; Euth, euthanasia.

\section{Discussion}

The causes underlying lung fibrogenesis remain largely unknown and are likely multifaceted, but exposure to certain irritants, including cigarette smoke, mineral dusts, and radiation, have been shown to induce redox perturbations and oxidative stress as well as pulmonary fibrosis $(10,28)$. Disruption of redox homeostasis, particularly in the epithelium, can promote acute lung injury and increased apoptosis (10,29), and PSSG has recently emerged as a key mediator of apoptosis and disease pathogenesis $(5,10)$. The present study demonstrates that both genetic ablation of Gstp and pharmacological inhibition of GSTP with the clinically relevant inhibitor, TLK117, attenuate increases in collagen content, $\alpha$-SMA immunoreactivity, FAS-SSG, and total PSSG in two different mouse models of lung fibrosis. GSTP immunoreactivity and an interaction between FAS and GSTP were also increased in the lungs of IPF patients, suggesting potential clinical relevance for our observations. For the first time to our knowledge, we have shown that expression and activity of GSTP can promote lung fibrogenesis and that pharmacological inhibition of this enzyme via the airways may represent a novel therapeutic strategy for the treatment of fibrotic lung disease. Further, these results underscore the importance of GSTP in PSSG, particularly FAS-SSG, and that GSTP may mediate lung fibrogenesis in part through this mechanism.

It is not entirely clear, based on our findings, in which cell types GSTP exerts its profibrotic role, given the widespread increases in GSTP immunoreactivity in lungs from mice with bleomycin- and AdTGF $\beta$-induced lung fibrosis (Figure 1D). Given that GSTP immunoreactivity is increased in lung tissue from patients with IPF in reactive type II epithelial cells within areas of active fibrosis and in pro-SPC-expressing cells within areas of bronchiolization (Figure 1B), it is plausible that GSTP-linked S-glutathionylation in type II alveolar epithelial cells contributes to disease pathogenesis. In this regard, chronic injury to type II cells and the loss of their normal restitution are considered important in the pathogenesis of $\operatorname{IPF}(30,31)$. In healthy lungs, GSTP is present in bronchial epithelial cells in which it has been implicated in the detoxification of inhaled pollutants (Figure 1D and ref. 32). Increases in GSTP immunoreactivity observed in reactive type II cells and areas of bronchiolization (Figure 1) may facilitate PSSG reactions that are important in cell death, including FAS-SSG $(5,10)$. Observations herein demonstrating that pharmacological inhibition or genetic ablation of GSTP resulted in less caspase- 3 and -8 activity and dampened FAS-SSG are consistent with a role of GSTP-linked S-glutathionylation in promoting alveolar type II epithelial cell death. It is, however, plausible that, besides FAS, other protein targets for GSTP-catalyzed S-glutathionylation relevant to lung fibrogenesis exist, and additional studies will be required to elucidate these targets.

The exact mechanisms whereby GSTP facilitates S-glutathionylation reactions are not fully known (33-35). GSTP functions as a ligandin, and its binding to target proteins affects their function. The identity of GSTP's target proteins in settings of fibrosis remains unclear, although we demonstrate herein an increased association between GSTP and FAS in mice with AdTGF $\beta$-induced fibrosis and in lung tissues from patients with IPF. It remains unclear whether the ability of GSTP to bind proteins controls subsequent S-glutathionylation reactions $(36,37)$. A recent study demonstrated that complex formation of GSTP1 with AMPK led to enzymatic activation of GSTP and, in turn, facilitated S-glutathionylation (38). We demonstrated herein that administration of TLK117 into airways attenuated FAS-SSG in association with a decreased interaction between GSTP and FAS (Figure 7, D and E). These data suggest that GSTP's ability to bind FAS is important in the catalysis of FAS-SSG. Cysteine 294 of mouse FAS was previously demonstrated to be the target of PSSG, and previous studies from our laboratory demonstrat- 
A

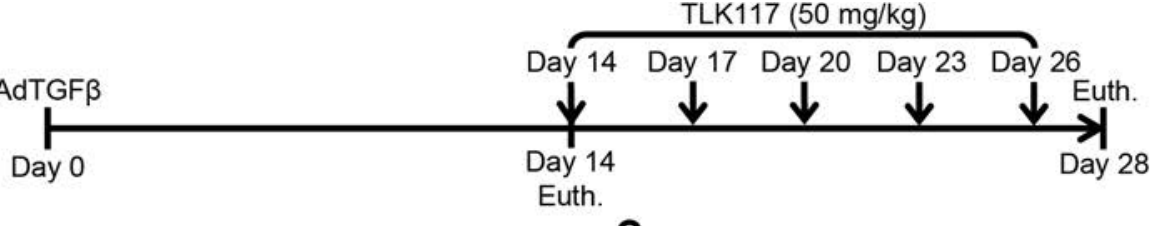

B

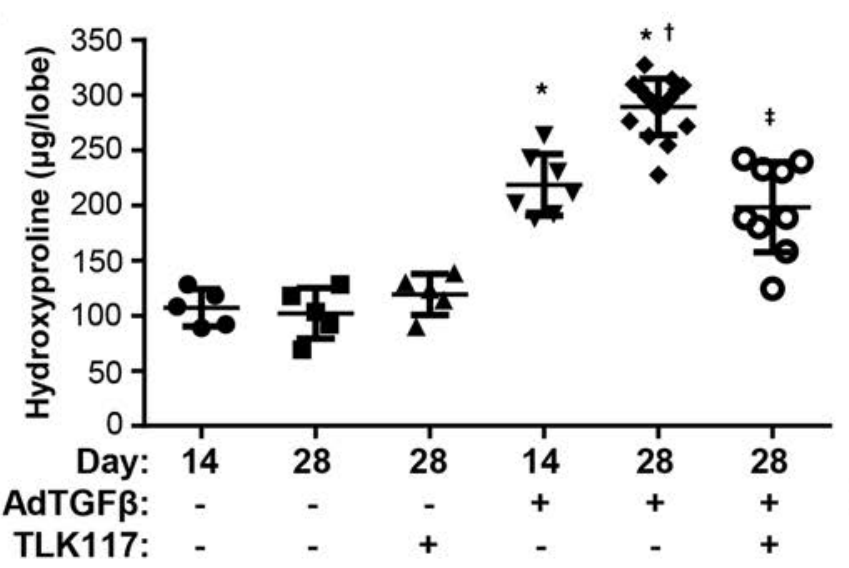

C

D
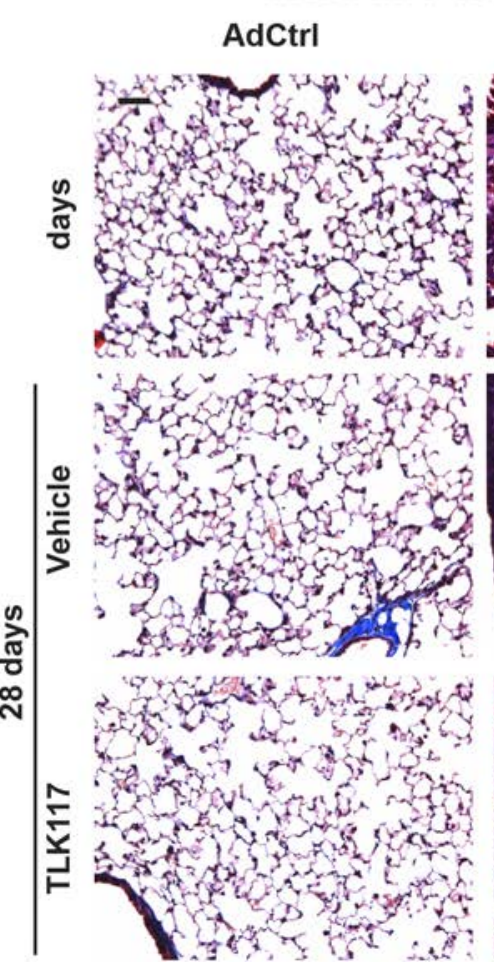

Masson's Trichrome

AdTGF $\beta$
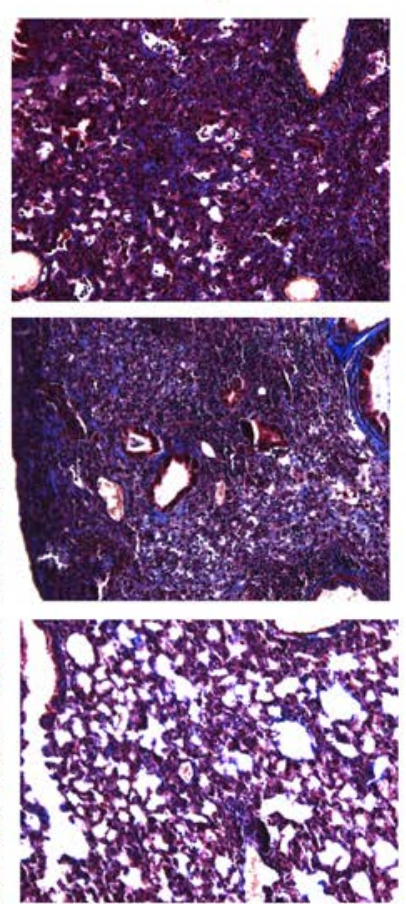

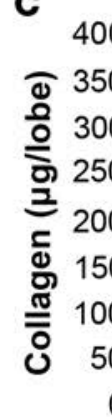
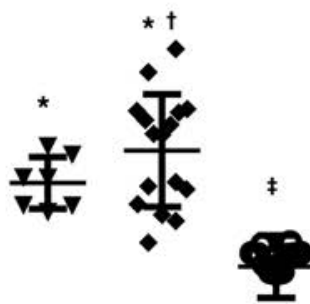

○

E $\alpha$-SMA $\begin{array}{rcccccc}\text { Day: } & 14 & 28 & 28 & 14 & 28 & 28 \\ \text { AdTGF } \beta: & - & - & - & + & + & + \\ \text { TLK117: } & - & - & + & - & - & +\end{array}$

AdTGF $\beta$

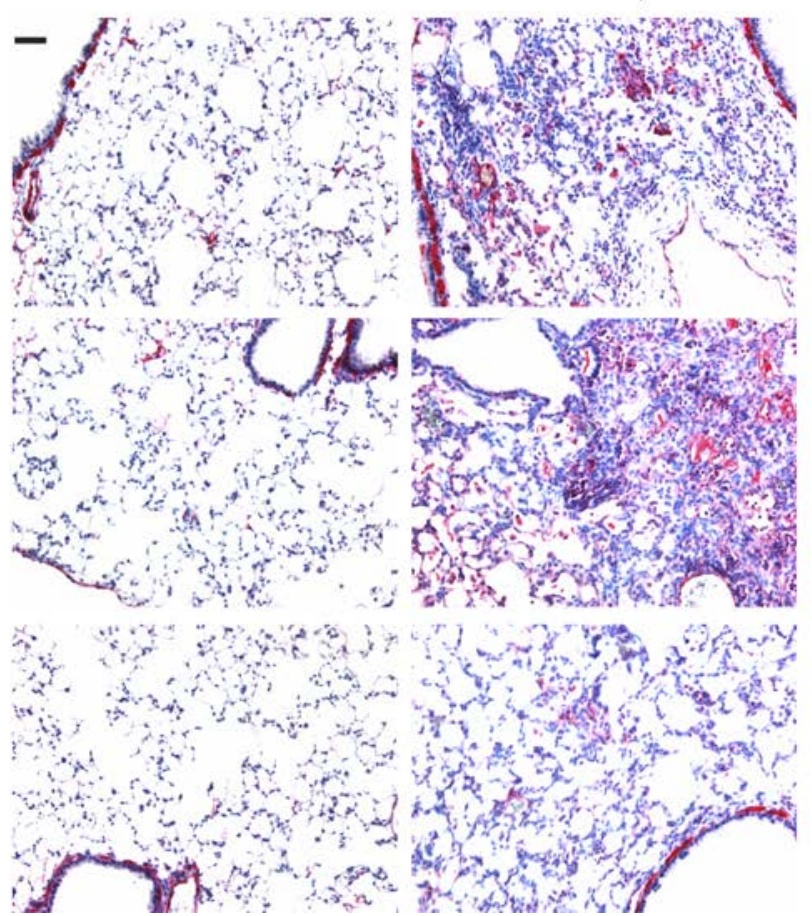

Figure 5. TLK117-mediated GSTP inhibition attenuates AdTGF $\beta$-induced collagen content. (A) Schematic of the TLK117 treatment strategy with the AdTCF $\beta$ model. ( $B$ and $\mathbf{C}$ ) Assessment of hydroxyproline and soluble collagen content in mouse lung homogenates. ${ }^{*} P<0.05$ relative to AdCtrl group, $\dagger P<0.05$ relative to 14 -day AdTGF $\beta$ group, $\ddagger P<0.05$ relative to 28 -day AdTGF $\beta$ /vehicle group by 1 -way ANOVA with a Tukey post-test. Shown are pooled data from 2 independent experiments ( $n=5-16$ per group). (D) Assessment of fibrotic remodeling by Masson's trichrome staining. (E) Assessment of $\alpha$-SMA immunoreactivity by immunohistochemistry (red, $\alpha$-SMA; blue, hematoxylin). Scale bar: $100 \mu$ m. AdCtrl, control adenovirus; AdTCF $\beta$, adenovirus expressing active TCF- $\beta 1 ; \alpha$-SMA, $\alpha$-smooth muscle actin; Euth, euthanization. 

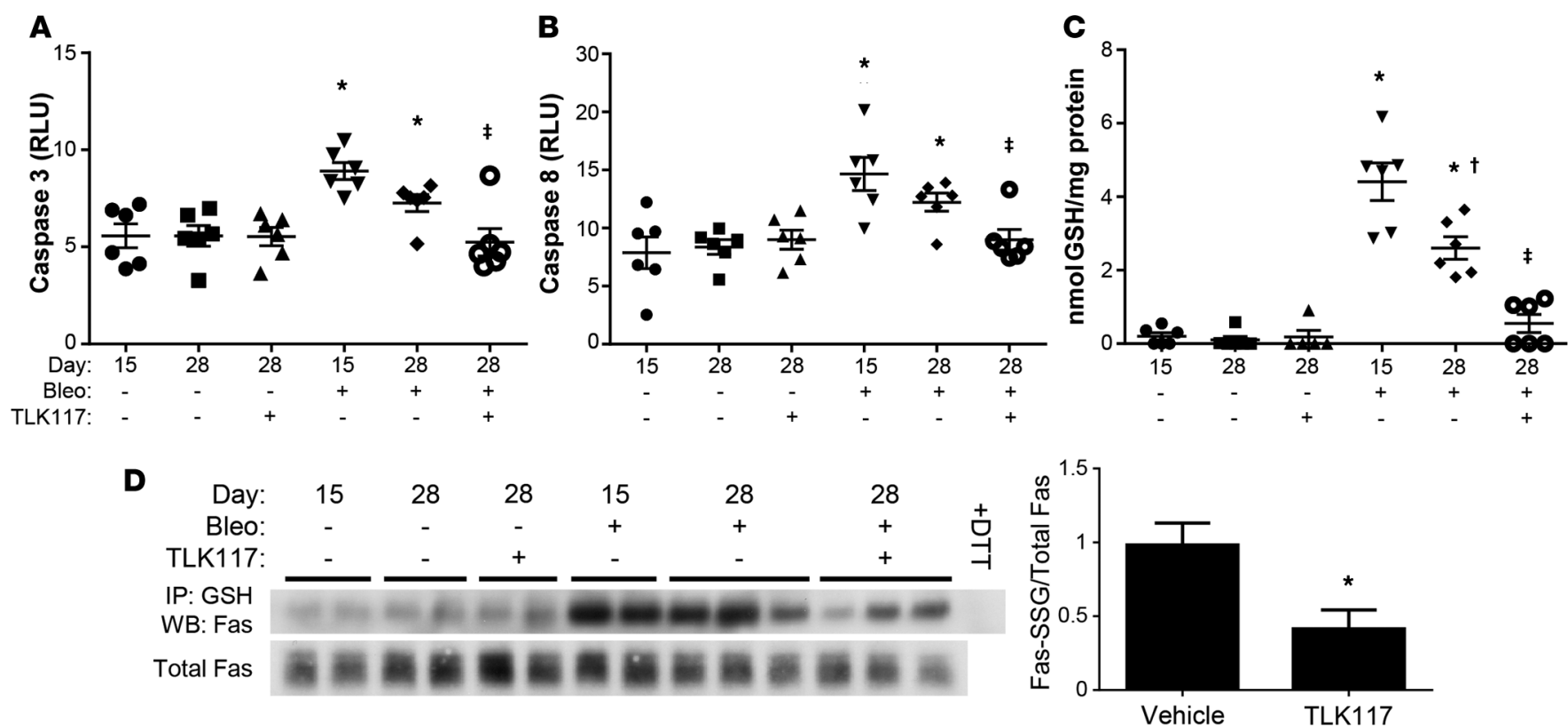

Figure 6. TLK117-mediated GSTP inhibition attenuates bleomycin-induced caspase activation and protein S-glutathionylation. Wild-type mice were treated with bleomycin and subsequently with TLK117, as described in Figure 4D. (A and B) Measurement of caspase-3 and -8 activity in mouse lungs homogenates. (C) Measurement of total PSSG with a modified DTNB assay. (A-C) ${ }^{*} P<0.05$ relative to PBS group, $+P<0.05$ relative to 15 -day bleomycin group, $¥ P<0.05$ relative to 28 -day bleomycin/vehicle group by 1-way ANOVA with a Tukey post-test. Shown are pooled data from 2 independent experiments ( $n=5-6$ per group). (D) Assessment of FAS-SSG by immunoprecipitation of total PSSG and Western blot for FAS. Significant differences between 28-day bleomycin-treated vehicle and TLK117 groups were assessed by densitometric analysis. ${ }^{*} P<0.05$ by 2-tailed unpaired Student's $t$ test ( $n=3$ per group). Data shown in $\mathbf{D}$ are representative of 1 of 2 independent experiments. FAS-SSG, S-glutathionylated FAS; GSH, glutathione; Bleo, bleomycin; DTT, dithiothreitol; WB, Western blot.

ed that formation of a sulfenic acid intermediate precedes GSTP-catalyzed S-glutathionylation of FAS in lung epithelial cells exposed to FASL (8). It remains unclear whether, in settings of lung fibrosis, a sulfenic acid residue of FAS is required for GSTP-catalyzed S-glutathionylation. The source of oxidants required for FAS-SSG also remains unclear. Previous studies have illuminated the importance of NOX4 activation as a source of hydrogen peroxide $\left(\mathrm{H}_{2} \mathrm{O}_{2}\right)$ in myofibroblasts and have indicated that release of $\mathrm{H}_{2} \mathrm{O}_{2}$ from fibroblasts is an important signal for apoptosis of epithelial cells (39-41). A prior study from our laboratory demonstrated that oxidative processing of latent FAS within the endoplasmic reticulum generates $\mathrm{H}_{2} \mathrm{O}_{2}$, which enables subsequent FAS-SSG (8). Further studies will therefore be required to identify the importance of $\mathrm{H}_{2} \mathrm{O}_{2}$ and the source of its production for GSTP-linked S-glutathionylation in settings of fibrosis. While FAS signaling is important for epithelial cell apoptosis, it has also been linked to myofibroblast activation, proliferation, and evasion from apoptosis $(6,9,42-44)$. Our current study did not determine whether GSTP and S-glutathionylation chemistry plays a role in myofibroblast behavior, which will require additional investigation. In addition to GSTP, it is possible that other GST isoforms may be involved in S-glutathionylation and pulmonary fibrosis as well. GSTM, GSTT, and GSTO polymorphisms have been linked to lung cancer, asthma, and COPD (45-49) and to PSSG (38). Further research will be required to elucidate the role other GSTs in S-glutathionylation and lung fibrosis. Despite the profibrogenic role of GSTP described in the present study, it is important to acknowledge that GSTP has important homeostatic functions. GSTP plays a prominent role in detoxification of the unsaturated aldehyde, acrolein (38). Ablation of Gstp has been shown to increase endothelial dysfunction in response to cigarette smoke, which contains high levels of acrolein (38). Similarly, absence of Gstp enhanced myocardial sensitivity to ischemia/reperfusion injury in association with increased acrolein-adducted proteins (49). Thus, the potential protective effect of inhibition of GSTP will likely be context specific.

Of significant clinical interest is the administration of the GSTP-specific inhibitor, TLK117. The development of TLK117 and its prodrug, TLK199, was prompted by the discovery that GSTP inhibition promotes myeloid cell hyperplasia by promoting c-Jun N-terminal kinase (JNK) activation by disrupting 


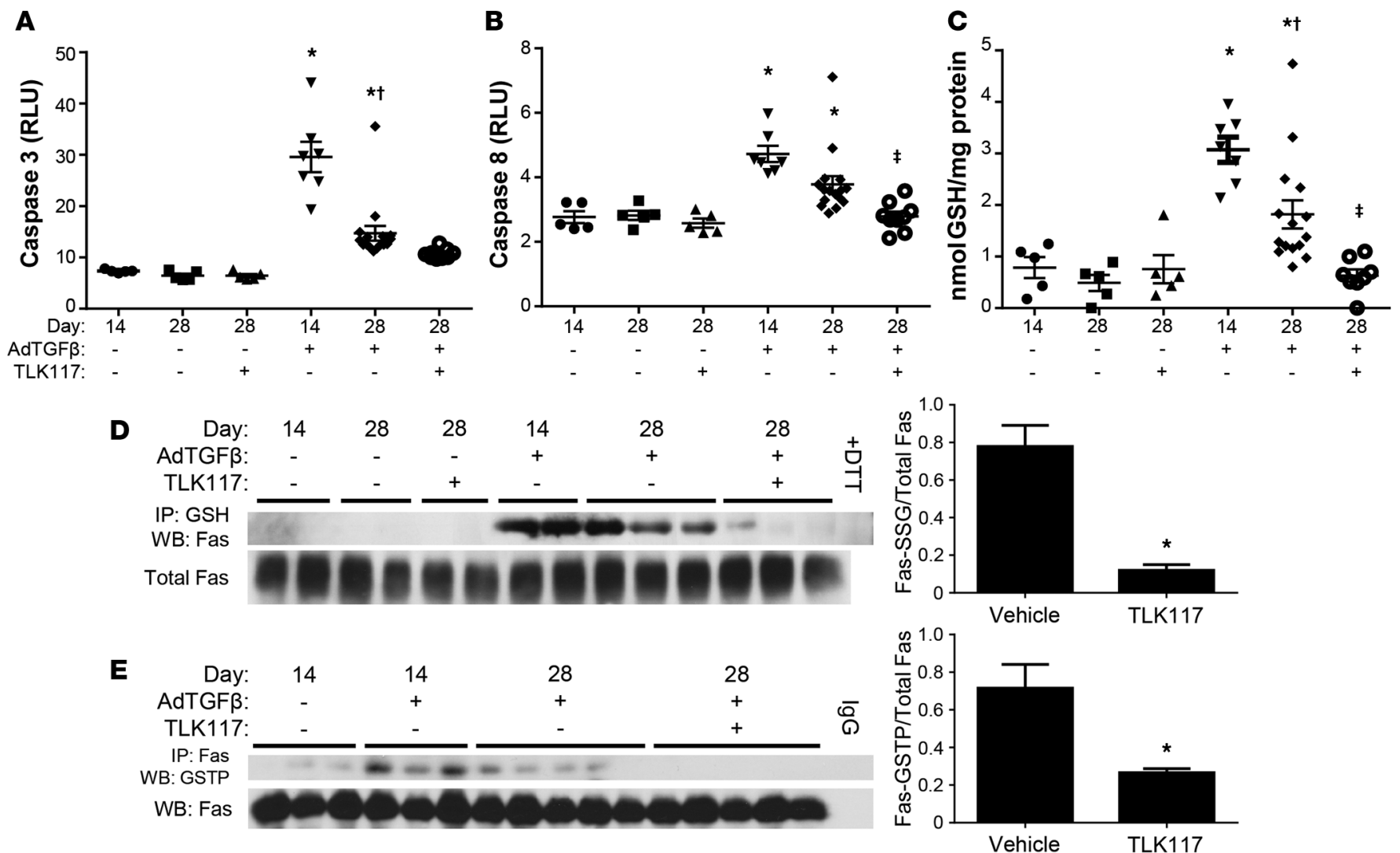

Figure 7. TLK117-mediated GSTP inhibition attenuates AdTCF $\beta$-induced caspase activation and protein S-glutathionylation. Wild-type mice were treated with AdTCF $\beta$ and subsequently with TLK117, as described in Figure 5A. (A and B) Measurement of caspase-3 and -8 activity in mouse lung homogenates. (C) Measurement of total PSSG with a modified DTNB assay. (A-C) ${ }^{*} P<0.05$ relative to AdCtrl group, $+P<0.05$ relative to 14 -day AdTCF $\beta$ group, $¥ P<0.05$ relative to 28 -day AdTGF $\beta$ /vehicle group by 1-way ANOVA with a Tukey post-test. Shown are pooled data from 2 independent experiments ( $n=5-16$ per group). (D) Assessment of FAS-SSG by immunoprecipitation of total PSSG and Western blot for FAS. (E) Assessment of FAS-GSTP interaction by immunoprecipitation of FAS from whole-lung homogenates and detection of GSTP by Western blot. Significant differences between 28-day AdTCF $\beta$-treated vehicle and TLK117 groups were assessed by densitometric analysis. ${ }^{*} P<0.05$ by 2-tailed unpaired Student's $t$ test ( $n=3$ per group for $\mathbf{D}$ and $n=3-5$ per group for $\mathbf{E})$. The data shown in $\mathbf{D}$ and $\mathbf{E}$ are representative of 1 of 2 independent experiments. GSTP, glutathione-S-transferase $\pi$; FAS-SSG, S-glutathionylated FAS; AdCtrl, control adenovirus; AdTGF $\beta$, adenovirus expressing active TCF- $\beta 1$; GSH, glutathione; DTT, dithiothreitol; WB, Western blot; IgG, immunoglobulin control.

GSTP-JNK interaction $(24,26,33,50)$. JNK signaling is an important mediator in lung inflammation and remodeling, and we have demonstrated a causal role for JNK1 in the development of pulmonary fibrosis (51-53). It is possible that TLK117-mediated inhibition of GSTP may also block lung fibrogenesis through a mechanism involving JNK signaling. Interestingly, the data presented in Figures 4 and 5 demonstrate that TLK117 may be capable not only of attenuating fibrosis progression, but also of reversing existing increases in collagen content, although additional studies will be necessary to elucidate the impact of TLK117 in additional models of persistent fibrosis, including age-associated pulmonary fibrosis. It is possible that TLK117-mediated GSTP inhibition stimulates collagen degradation through upregulation or activation of collagenases. Intriguingly, the JNK pathway has been shown to regulate matrix metalloproteinases $(54,55)$, which are essential for collagen clearance and reepithelialization during wound healing (56). Future studies will therefore be aimed at obtaining mechanistic insights into how GSTP-linked PSSG affects matrix degrading pathways and the subsequent impact on fibrotic remodeling in the lung.

Bleomycin-induced fibrosis, FAS-SSG, and caspase activation in mouse lungs are all abundant about 2 weeks after treatment $(8,18)$. Therefore, administration of TLK117 at this time was chosen to address the impact of TLK117 in settings of existing fibrosis. Controversy exists regarding the clinical relevance of bleomycin as a model of IPF, largely because its mechanism of action is not fully known and because bleomycin-induced lung fibrosis resolves in adult mice $(18,57)$. The observations herein, however, demonstrate a similar protective impact of genetic ablation or inhibition of GSTP in the AdTGF $\beta$ model of fibrosis, 
which is believed to induce persistent remodeling (7). The demonstration of a protective effect of TLK117 or GSTP ablation in both models, along with the demonstration of increases in GSTP and associations between GSTP and FAS in lung tissues from patients with IPF, collectively points to a putative causal role of GSTP-linked S-glutathionylation in patients with IPF.

Clinical trials utilizing TLK199 have shown that this compound is well-tolerated and exerts only minor gastrointestinal side effects (23), which may be anticipated given the abundance of GSTP in the stomach and intestinal epithelium $(32,58)$. Patients exposed to high doses of TLK199 also exhibited an increased incidence and severity of abnormal skin odor (23). The dosing regimen of TLK199 in clinical trials, however, was either intravenous or oral rather than via the airways $(22,23)$. Further, given the short half-life of this drug when administered via intravenous or oral routes, patients were given daily doses of up to 4,500 mg to exert therapeutic effects (23). It is possible that administration of active TLK117 or the prodrug, TLK199, via inhalation could also have a beneficial effect in patients with IPF, and such a dosing regimen would be an attractive strategy to directly target GSTP-expressing epithelial cells. However, additional clinical testing with these compounds using an inhalation regimen will be required to establish the feasibility of this approach in patients.

New therapies for pulmonary fibrosis have been the subject of much research and intense pharmaceutical development in recent years. Corticosteroids and immunosuppressants have routinely demonstrated to be of limited efficacy and to elicit potent side effects in IPF patients (59-62). Pirfenidone and nintedanib, the most promising recent additions to this repertoire, have modest effects on lung function decline (6365). Antioxidants have been proposed as potential therapeutics for fibrotic lung diseases, particularly the GSH precursor, N-acetylcysteine (66). However, a recent phase III clinical trial demonstrated no beneficial effects of $\mathrm{N}$-acetylcysteine on IPF progression or lung function decline (4), although a pharmacogenomic interaction involving TOLLIP and MUC5B SNPs and a response of IPF patients to N-acetylcysteine has become apparent (67). The current study points to the importance not of GSH per se, but a unique facet of GSH chemistry, which involves its covalent incorporation into proteins, catalyzed by GSTP, a biochemical pathway that has not previously been recognized in settings of pulmonary fibrosis. Thus, a clinical trial utilizing the GSTP inhibitor described herein in IPF patients appears well warranted and may yield new insights into the functional importance of S-glutathionylation chemistry in the pathogenesis and progression of this deadly disease.

\section{Methods}

Additional details are available in the Supplemental Methods.

Study design. The aim of this study was to determine the effects of Gstp knockout and GSTP inhibition on two mouse models of lung fibrosis (bleomycin and AdTGF $\beta$ ). All studies were age and sex matched, and each experiment was conducted at least twice with a minimum of 8 mice per group based on experiments conducted previously $(8,51-53)$. Endpoints, including changes in collagen content, $\alpha$-SMA immunoreactivity, FAS-SSG, total PSSG, and FAS-GSTP interaction, between 2 and 4 weeks were selected based on previously reported findings $(7,8,18)$. GSTP expression and FAS-GSTP interaction were also measured in human IPF lung samples relative to healthy controls, and at least 10 samples per group were analyzed. The effects of Gstp and Fas deficiency ( $L p r$ genotype) and reconstitution on caspase activity were also measured in lung fibroblasts in vitro; each in vitro experiment was conducted at least twice, each time in triplicate. All data collected for each experiment is represented in the figures, and no outliers were excluded.

Human lung samples. Deidentified human lung samples from both normal and IPF patients were obtained from the Lung Tissue Research Consortium (LTRC).

Mice. Male and female C57BL/6NJ mice, both wild-type and deficient for both Gstp1 and 2 (Gstp ${ }^{-/}$) $(68,69)$, were bred at the University of Vermont and used at 8 weeks of age. Mice were treated with either bleomycin $(0.125 \mathrm{U} /$ mouse $)$ or AdTGF $\beta\left(5 \times 10^{8} \mathrm{pfu} /\right.$ mouse $)$ oropharyngeally to induce lung fibrotic remodeling. PBS and an adenovirus expressing an empty vector (AdCtrl) were used as controls, respectively. In the bleomycin model, mice were euthanized at either day 15 or 28. In the AdTGF $\beta$ model, mice were euthanized at day 21. Right lung lobes were frozen for detection of collagen content, FAS-SSG, total PSSG, FAS-GSTP interaction, mRNA production, and protein expression. Left lobes were fixed in $4 \%$ paraformaldehyde in PBS, embedded in paraffin, and sectioned for immunohistochemical analysis.

Cells. Primary normal lung fibroblasts from both wild-type and $\mathrm{Lpr}$ mice, and mouse type II alveolar epithelial (C10) cells, were obtained and cultured as previously described (8). Lpr fibroblasts were transfect- 
ed with constructs expressing either wild-type or C294A mutant Fas using Lipofectamine LTX with PLUS reagent according to the manufacturer's protocol (A21621; Invitrogen). pcDNA3.1 was used as a control. Wild-type fibroblasts remained untransfected throughout the experiment. Fibroblasts and type II cells were pretreated with $50 \mu \mathrm{M}$ of the active GSTP inhibitor, TLK117, in serum-free media for 2 hours and subsequently with $500 \mathrm{ng} / \mathrm{ml}$ of FASL (ALX-522-001; Enzo Life Sciences) with $1 \mu \mathrm{g} / \mathrm{ml}$ of anti-FLAG crosslinking antibody (M2; F1804; Sigma-Aldrich) for 4 hours as previously described (8). M2 only was used as a control. Type II cells were also treated with $10 \mathrm{ng} / \mathrm{ml}$ mouse TNF- $\alpha$ (GF027; EMD Millipore) and $5 \mu \mathrm{g} /$ $\mathrm{ml}$ cycloheximide (Sigma-Aldrich) for 4 hours. Cells were then lysed in a NP-40 lysis buffer supplemented with protease and phosphatase inhibitors to collect protein.

Synthesis of TLK117. TLK117 [ $\gamma$-glutamyl-S-(benzyl)cysteinyl-R-(-)-phenyl glycine] was generated by Fmoc solid-state peptide synthesis with HBTU as previously described (8) and was confirmed to be $>98 \%$ pure by HPLC. TLK 117 was administered oropharyngeally at a dose of $50 \mathrm{mg} / \mathrm{kg}$ in a $0.375 \mathrm{M}$ Tris- $\mathrm{HCl}$ solution, $\mathrm{pH}=7.4$, with $0.02 \%$ DMSO. This Tris- $\mathrm{HCl} / \mathrm{DMSO}$ solution was used as a vehicle control. 50 $\mathrm{mg} / \mathrm{kg}$ was selected based on the data presented in Figure 6A. Treatments were performed once every 3 days from day 14 to day 26 in both bleomycin and AdTGF $\beta$ models for a total of 5 treatments. Mice that were euthanized at day 15 in the bleomycin model and day 14 in the AdTGF $\beta$ model did not receive either TLK117 or the vehicle control.

Detection of FAS-GSTP interaction. Human lung samples obtained from the LTRC were homogenized in an NP-40 lysis buffer containing 10\% glycerol and protease and phosphatase inhibitors as previously described (8). FAS was isolated by immunoprecipitation with an anti-FAS antibody (554254; BD Biosciences) and recombinant protein G agarose beads (15920-010; Invitrogen), and GSTP was detected by Western blot with a GSTP-specific antibody (312; MBL International).

Detection of FAS-SSG. Mouse lungs were homogenized in a Tris/SDS lysis buffer, $\mathrm{pH}=7.4$, supplemented with protease and phosphatase inhibitors, $20 \mathrm{mM} \mathrm{N}$-ethylmaleimide, $100 \mu \mathrm{M}$ diethylene triamine pentaacetic acid, and $100 \mu \mathrm{M}$ neocuproine (Sigma-Aldrich). PSSGs were isolated from mouse lung homogenates by immunoprecipitation with a PSSG-specific antibody (101-A; ViroGen) and recombinant protein $\mathrm{G}$ agarose beads (Invitrogen) as previously described (10). As a control, lysate was pretreated with $50 \mu \mathrm{M}$ DTT to reduce PSSG also as previously described (10). Both total FAS and FAS-SSG content was assessed by Western blot with an anti-FAS antibody (05-351; EMD Millipore).

Detection of total PSSG content. Total PSSG content was detected in mouse lungs with a modified DTNB assay as previously described $(8,70)$.

GSTP activity assay. Mouse lungs were homogenized in a $100 \mathrm{mM}$ potassium phosphate buffer, $\mathrm{pH}=$ 6.5, with protease and phosphatase inhibitors and $0.1 \%$ Triton X-100. GSTP was isolated from homogenates by immunoprecipitation with a GSTP-specific antibody (MBL International) and recombinant protein $\mathrm{G}$ agarose beads (Invitrogen) as previously described (8). Normal rabbit IgG was used as a control. Beads were washed and resuspended in $100 \mathrm{mM}$ potassium phosphate buffer, $\mathrm{pH}=6.5$, with $1 \mathrm{mM}$ EDTA, and GSTP activity was assessed with a modified CDNB assay as previously described (8). Results are expressed as nmol substrate per mg protein per minute, wherein one unit represents the consumption of substrate by $1 \mathrm{mg}$ of GSTP per minute.

Caspase activity. Caspase- 3 and -8 activity was measured with Caspase-Glo activity reagents (G8091 and G8201; Promega) according to the manufacturer's instructions.

Assessment of collagen content. Hydroxyproline content was assessed in the right upper lobe of the lung as previously described (71). Lobes were digested overnight in $6 \mathrm{~N} \mathrm{HCl}$ and mixed with chloramine-T for 20 minutes and with perchloric acid for 5 minutes at room temperature. Ehrlich's reagent was added for 20 minutes at $60^{\circ} \mathrm{C}$, and absorbance was measured at $561 \mathrm{~nm}$. All reagents were obtained from Sigma-Aldrich. Soluble collagen was assessed from the right middle lobe of the lung as previously described (72). Lobes were digested overnight with $10 \mathrm{mg}$ pepsin in $0.5 \mathrm{M}$ acetic acid, and collagen content was assessed with a Sircol collagen assay kit according to the manufacturer's instructions (S1000; Biocolor Life Science).

Tissue staining and immunohistochemistry. Fibrotic remodeling was assessed in mouse lung sections by Masson's trichrome staining. For immunohistochemical analysis, human lung sections from the LTRC were probed with antibodies specific for GSTP (MBL International) and pro-SPC (AB3786; EMD Millipore). Mouse lung sections were probed for either GSTP (AP9199c; Abgent) or $\alpha$-SMA (A2547; Sigma-Aldrich). All sections were developed with a universal Vectastain ABC-AP kit (AK-5200; Vector Laboratories) and a Vector Red AP substrate kit (SK-5100; Vector Laboratories) and were counterstained with hematoxylin. 
Slides were subsequently coverslipped, viewed, and photographed as previously described (8). Each scale bar represents either 50 or $100 \mu \mathrm{m}$ as indicated.

Western blotting. Protein concentration in whole lung homogenates was determined using a DC protein assay (Bio-Rad), and $25 \mu \mathrm{g}$ of total protein was separated via $8 \%$ SDS-PAGE, transferred onto Immobilon-P membrane (IPVH00010; EMD Millipore), and blocked with 5\% BSA in TBS with 0.1\% Tween 20. The mouse $\beta$-actin antibody was purchased from Sigma-Aldrich (A5441). Protein was visualized using an ECL Western blotting substrate (32106; Thermo Fisher Scientific) and developed on blue X-ray film (F-BX810; Phenix Research Products) with a Summit QCP X-ray film processor. Densitometric analysis was performed with Discovery Series Quantity One 1-D analysis software (Bio-Rad).

Statistics. Statistical analysis was performed with GraphPad Prism v6.0 (GraphPad Software). All results are presented as the mean \pm SEM. Statistically significant differences were assessed with a 1-way ANOVA with a Tukey post-test, a 2-way ANOVA with a Tukey post-test, or a 2-tailed unpaired Student's $t$ test where appropriate. A $P$ value of less than 0.05 was considered significant.

Study approval. The Institutional Review Board of the University of Vermont has determined that the use of the deidentified human samples used in this study does not constitute human subjects research as recognized by 45 CFR 46.102(f) and the US Department of Health and Human Services Office for Human Research Protections Guidance on Research Involving Coded Private Information or Biological Specimens. All studies involving mice were approved by the Institutional Animal Care and Use Committee at the University of Vermont.

\section{Author contributions}

DHM, JLJV, VA, and YMWJH prepared this manuscript, planned experiments, and analyzed data. DHM, KGL, RWS, XQ, MSI, JDN, SA, DTC, and VA conducted experiments and analyzed data. SF synthesized the TLK117 used throughout our studies. CJH and CRW provided the Gstp ${ }^{-1-}$ mice. KDT, DMT, KJB, DJT, RCB, CGI, AVDV, and VA all provided crucial guidance, resources, and facilities necessary to complete this project.

\section{Acknowledgments}

This research is funded by NIH grants T32 HL076122, R01 HL079331, R01 HL122383, and F32 HL124902. We thank Page Spiess for her technical assistance.

Address correspondence to: Yvonne M.W. Janssen-Heininger, Department of Pathology and Laboratory Medicine, University of Vermont Medical Center, 149 Beaumont Avenue, HSRF 216A, Burlington, Vermont 05405, USA. Phone: 802.656.0995; E-mail: yvonne.janssen@uvm.edu.

1. Lomas NJ, Watts KL, Akram KM, Forsyth NR, Spiteri MA. Idiopathic pulmonary fibrosis: immunohistochemical analysis provides fresh insights into lung tissue remodelling with implications for novel prognostic markers. Int J Clin Exp Pathol. 2012;5(1):58-71.

2. Blackwell TS, et al. Future directions in idiopathic pulmonary fibrosis research. Am J Respir Crit Care Med. 2014;189(2):214-222.

3. Raghu G, Weycker D, Edelsberg J, Bradford WZ, Oster G. Incidence and prevalence of idiopathic pulmonary fibrosis. Am J Respir Crit Care Med. 2006;174(7):810-816.

4. Idiopathic Pulmonary Fibrosis Clinical Research Network, Martinez FJ, de Andrade JA, Anstrom KJ, King TE Jr, Raghu G. Randomized trial of acetylcysteine in idiopathic pulmonary fibrosis. N Engl J Med. 2014;370(22):2093-2101.

5. Janssen-Heininger YM, et al. Regulation of apoptosis through cysteine oxidation: implications for fibrotic lung disease. Ann $N Y$ Acad Sci. 2010;1203:23-28.

6. Wynes MW, et al. Increased cell surface FAS expression is necessary and sufficient to sensitize lung fibroblasts to FAS ligation-induced apoptosis: implications for fibroblast accumulation in idiopathic pulmonary fibrosis. J Immunol. 2011;187(1):527537.

7. Sime PJ, Xing Z, Graham FL, Csaky KG, Gauldie J. Adenovector-mediated gene transfer of active transforming growth factor-beta1 induces prolonged severe fibrosis in rat lung. J Clin Invest. 1997;100(4):768-776.

8. Anathy V, et al. Oxidative processing of latent FAS in the endoplasmic reticulum controls the strength of apoptosis. $\mathrm{Mol}$ Cell Biol. 2012;32(17):3464-3478.

9. Golan-Gerstl R, Wallach-Dayan SB, Amir G, Breuer R. Epithelial cell apoptosis by FAS ligand-positive myofibroblasts in lung fibrosis. Am J Respir Cell Mol Biol. 2007;36(3):270-275.

10. Anathy V, Roberson EC, Guala AS, Godburn KE, Budd RC, Janssen-Heininger YM. Redox-based regulation of apoptosis: S-glutathionylation as a regulatory mechanism to control cell death. Antioxid Redox Signal. 2012;16(6):496-505.

11. Cantin AM, Hubbard RC, Crystal RG. Glutathione deficiency in the epithelial lining fluid of the lower respiratory tract in idio- 
pathic pulmonary fibrosis. Am Rev Respir Dis. 1989;139(2):370-372.

12. Anathy V, et al. Redox amplification of apoptosis by caspase-dependent cleavage of glutaredoxin 1 and S-glutathionylation of FAS. J Cell Biol. 2009;184(2):241-252.

13. Mailloux RJ, et al. Glutaredoxin-2 is required to control oxidative phosphorylation in cardiac muscle by mediating deglutathionylation reactions. J Biol Chem. 2014;289(21):14812-14828.

14. Sheehan D, Meade G, Foley VM, Dowd CA. Structure, function and evolution of glutathione transferases: implications for classification of non-mammalian members of an ancient enzyme superfamily. Biochem J. 2001;360(pt 1):1-16.

15. Laborde E. Glutathione transferases as mediators of signaling pathways involved in cell proliferation and cell death. Cell Death Differ. 2010;17(9):1373-1380.

16. Zhou J, et al. Glutathione transferase P1: an endogenous inhibitor of allergic responses in a mouse model of asthma. Am J Respir Crit Care Med. 2008;178(12):1202-1210.

17. Feng X, Zheng BS, Shi JJ, Qian J, He W, Zhou HF. Association of glutathione S-transferase P1 gene polymorphism with the susceptibility of lung cancer. Mol Biol Rep. 2012;39(12):10313-10323.

18. Izbicki G, Segel MJ, Christensen TG, Conner MW, Breuer R. Time course of bleomycin-induced lung fibrosis. Int J Exp Pathol. 2002;83(3):111-119

19. Zhang HY, Gharaee-Kermani M, Zhang K, Karmiol S, Phan SH. Lung fibroblast alpha-smooth muscle actin expression and contractile phenotype in bleomycin-induced pulmonary fibrosis. Am J Pathol. 1996;148(2):527-537.

20. Tew KD, Manevich Y, Grek C, Xiong Y, Uys J, Townsend DM. The role of glutathione S-transferase P in signaling pathways and S-glutathionylation in cancer. Free Radic Biol Med. 2011;51(2):299-313.

21. Aesif SW, et al. In situ analysis of protein S-glutathionylation in lung tissue using glutaredoxin-1-catalyzed cysteine derivatization. Am J Pathol. 2009;175(1):36-45.

22. Raza A, et al. Phase 1-2a multicenter dose-escalation study of ezatiostat hydrochloride liposomes for injection (Telintra, TLK199), a novel glutathione analog prodrug in patients with myelodysplastic syndrome. J Hematol Oncol. $2009 ; 2: 20$.

23. Raza A, et al. A phase 2 randomized multicenter study of 2 extended dosing schedules of oral ezatiostat in low to intermediate-1 risk myelodysplastic syndrome. Cancer. 2012;118(8):2138-2147.

24. Adler V, et al. Regulation of JNK signaling by GSTp. EMBO J. 1999;18(5):1321-1334.

25. Morgan AS, Ciaccio PJ, Tew KD, Kauvar LM. Isozyme-specific glutathione S-transferase inhibitors potentiate drug sensitivity in cultured human tumor cell lines. Cancer Chemother Pharmacol. 1996;37(4):363-370

26. Ruscoe JE, et al. Pharmacologic or genetic manipulation of glutathione S-transferase P1-1 (GSTpi) influences cell proliferation pathways. J Pharmacol Exp Ther. 2001;298(1):339-345.

27. Oakley AJ, et al. The structures of human glutathione transferase P1-1 in complex with glutathione and various inhibitors at high resolution. J Mol Biol. 1997;274(1):84-100.

28. Camelo A, Dunmore R, Sleeman MA, Clarke DL. The epithelium in idiopathic pulmonary fibrosis: breaking the barrier. Front Pharmacol. 2014;4:173.

29. Araya J, Nishimura SL. Fibrogenic reactions in lung disease. Annu Rev Pathol. 2010;5:77-98.

30. Sisson TH, et al. Targeted injury of type II alveolar epithelial cells induces pulmonary fibrosis. Am J Respir Crit Care Med. 2010;181(3):254-263

31. Zoz DF, Lawson WE, Blackwell TS. Idiopathic pulmonary fibrosis: a disorder of epithelial cell dysfunction. Am J Med Sci. 2011;341(6):435-438.

32. Henderson CJ, McLaren AW, Wolf CR. In vivo regulation of human glutathione transferase GSTP by chemopreventive agents. Cancer Res. 2014;74(16):4378-4387.

33. Tew KD. Redox in redux: Emergent roles for glutathione S-transferase P (GSTP) in regulation of cell signaling and S-glutathionylation. Biochem Pharmacol. 2007;73(9):1257-1269.

34. Townsend DM, Manevich Y, He L, Hutchens S, Pazoles CJ, Tew KD. Novel role for glutathione S-transferase pi. J Biol Chem. 2009;284(1):436-445.

35. Zhou S, Lien YC, Shuvaeva T, DeBolt K, Feinstein SI, Fisher AB. Functional interaction of glutathione S-transferase pi and peroxiredoxin 6 in intact cells. Int J Biochem Cell Biol. 2013;45(2):401-407.

36. Manevich Y, Hutchens S, Tew KD, Townsend DM. Allelic variants of glutathione S-transferase P1-1 differentially mediate the peroxidase function of peroxiredoxin VI and alter membrane lipid peroxidation. Free Radic Biol Med. 2013;54:62-70.

37. Zhang J, Grek C, Ye ZW, Manevich Y, Tew KD, Townsend DM. Pleiotropic functions of glutathione S-transferase P. Adv Cancer Res. 2014;122:143-175.

38. Conklin DJ, Haberzettl P, Prough RA, Bhatnagar A. Glutathione-S-transferase P protects against endothelial dysfunction induced by exposure to tobacco smoke. Am J Physiol Heart Circ Physiol. 2009;296(5):H1586-H1597.

39. Hecker L, et al. Reversal of persistent fibrosis in aging by targeting Nox4-Nrf2 redox imbalance. Sci Transl Med. 2014;6(231):231ra247.

40. Hecker L, et al. NADPH oxidase-4 mediates myofibroblast activation and fibrogenic responses to lung injury. Nat Med. 2009;15(9):1077-1081.

41. Thannickal VJ. Mechanisms of pulmonary fibrosis: role of activated myofibroblasts and NADPH oxidase. Fibrogenesis Tissue Repair. 2012;5(suppl 1):S23

42. Golan-Gerstl R, Wallach-Dayan SB, Zisman P, Cardoso WV, Goldstein RH, Breuer R. Cellular FLICE-like inhibitory protein deviates myofibroblast FAS-induced apoptosis toward proliferation during lung fibrosis. Am J Respir Cell Mol Biol. 2012;47(3):271-279.

43. Kuwano K, et al. Essential roles of the FAS-FAS ligand pathway in the development of pulmonary fibrosis. J Clin Invest. 1999;104(1):13-19.

44. Wallach-Dayan SB, et al. Cutting edge: FasL(+) immune cells promote resolution of fibrosis. J Autoimmun. 2015;59:67-76

45. Demir A, et al. The role of GSTM1 gene polymorphisms in lung cancer development in Turkish population. J Carcinog. 2007;6:13.

46. Lima CS, Neri IA, Lourenco GJ, Faria IC, Ribeiro JD, Bertuzzo CS. Glutathione S-transferase mu 1 (GSTM1) and theta 1 
(GSTT1) genetic polymorphisms and atopic asthma in children from Southeastern Brazil. Genet Mol Biol. 2010;33(3):438-441.

47. Reszka E, Wasowicz W. Significance of genetic polymorphisms in glutathione S-transferase multigene family and lung cancer risk. Int J Occup Med Environ Health. 2001;14(2):99-113.

48. Harju TH, et al. Glutathione S-transferase omega in the lung and sputum supernatants of COPD patients. Respir Res. 2007;8:48.

49. Conklin DJ, et al. Genetic deficiency of glutathione S-transferase P increases myocardial sensitivity to ischemia-reperfusion injury. Circ Res. 2015;117(5):437-449.

50. Elsby R, et al. Increased constitutive c-Jun N-terminal kinase signaling in mice lacking glutathione S-transferase Pi. J Biol Chem. 2003;278(25):22243-22249.

51. Alcorn JF, van der Velden J, Brown AL, McElhinney B, Irvin CG, Janssen-Heininger YM. c-Jun N-terminal kinase 1 is required for the development of pulmonary fibrosis. Am J Respir Cell Mol Biol. 2009;40(4):422-432.

52. van der Velden JL, et al. Absence of c-Jun NH2-terminal kinase 1 protects against house dust mite-induced pulmonary remodeling but not airway hyperresponsiveness and inflammation. Am J Physiol Lung Cell Mol Physiol. 2014;306(9):L866-L875.

53. Velden JL, Alcorn JF, Guala AS, Badura EC, Janssen-Heininger YM. c-Jun N-terminal kinase 1 promotes transforming growth factor-beta1-induced epithelial-to-mesenchymal transition via control of linker phosphorylation and transcriptional activity of Smad3. Am J Respir Cell Mol Biol. 2011;44(4):571-581.

54. Ispanovic E, Haas TL. JNK and PI3K differentially regulate MMP-2 and MT1-MMP mRNA and protein in response to actin cytoskeleton reorganization in endothelial cells. Am J Physiol Cell Physiol. 2006;291(4):C579-C588.

55. Cheng CY, Hsieh HL, Hsiao LD, Yang CM. PI3-K/Akt/JNK/NF-kappaB is essential for MMP-9 expression and outgrowth in human limbal epithelial cells on intact amniotic membrane. Stem Cell Res. 2012;9(1):9-23.

56. Stevens LJ, Page-McCaw A. A secreted MMP is required for reepithelialization during wound healing. Mol Biol Cell. 2012;23(6):1068-1079.

57. Moeller A, Ask K, Warburton D, Gauldie J, Kolb M. The bleomycin animal model: a useful tool to investigate treatment options for idiopathic pulmonary fibrosis? Int J Biochem Cell Biol. 2008;40(3):362-382.

58. Conklin DJ, Haberzettl P, Lesgards JF, Prough RA, Srivastava S, Bhatnagar A. Increased sensitivity of glutathione S-transferase P-null mice to cyclophosphamide-induced urinary bladder toxicity. J Pharmacol Exp Ther. 2009;331(2):456-469.

59. Gross TJ, Hunninghake GW. Idiopathic pulmonary fibrosis. N Engl J Med. 2001;345(7):517-525.

60. Richeldi L, Davies HR, Ferrara G, Franco F. Corticosteroids for idiopathic pulmonary fibrosis. Cochrane Database Syst. 2003;(3):CD002880

61. Lynch JP. Corticosteroids in idiopathic pulmonary fibrosis. Curr Opin Pulm Med. 2001;7(5):298-308.

62. Walter N, Collard HR, King TE Jr. Current perspectives on the treatment of idiopathic pulmonary fibrosis. Proc Am Thorac Soc 2006;3(4):330-338.

63. King TE. A phase 3 trial of pirfenidone in patients with idiopathic pulmonary fibrosis. N Engl J Med. 2014;370(22):2083-2092

64. Ogura T, et al. Safety and pharmacokinetics of nintedanib and pirfenidone in idiopathic pulmonary fibrosis. Eur Respir $J$. 2015;45(5):1382-1392.

65. Richeldi L, et al. Efficacy and safety of nintedanib in idiopathic pulmonary fibrosis. N Engl J Med. 2014;370(22):2071-2082

66. Day BJ. Antioxidants as potential therapeutics for lung fibrosis. Antioxid Redox Signal. 2008;10(2):355-370.

67. Oldham JM, et al. TOLLIP, MUC5B, and the response to $\mathrm{N}$-acetylcysteine among individuals with idiopathic pulmonary fibrosis. Am J Respir Crit Care Med. 2015;192(12):1475-1482.

68. Henderson CJ, Smith AG, Ure J, Brown K, Bacon EJ, Wolf CR. Increased skin tumorigenesis in mice lacking pi class glutathione S-transferases. Proc Natl Acad Sci U S A. 1998;95(9):5275-5280.

69. Henderson CJ, Wolf CR. Knockout and transgenic mice in glutathione transferase research. Drug Metab Rev. 2011;43(2):152164

70. Rahman I, Kode A, Biswas SK. Assay for quantitative determination of glutathione and glutathione disulfide levels using enzymatic recycling method. Nat Protoc. 2006;1(6):3159-3165

71. Woessner JF Jr. The determination of hydroxyproline in tissue and protein samples containing small proportions of this imino acid. Arch Biochem Biophys. 1961;93:440-447.

72. Tully JE, et al. Epithelial NF- $\mathrm{BB}$ orchestrates house dust mite-induced airway inflammation, hyperresponsiveness, and fibrotic remodeling. J Immunol. 2013;191(12):5811-5821. 